\title{
Review \\ Effects of Different Trunk Training Methods for Chronic Low Back Pain: A Meta-Analysis
}

\author{
Dhananjaya Sutanto $^{1}{ }^{\mathbb{D}}$, Robin S. T. Ho ${ }^{1}$, Eric T. C. Poon ${ }^{2}$, Yijian Yang ${ }^{1}{ }^{\mathbb{C}}$ and Stephen H. S. Wong ${ }^{1, *(\mathbb{D})}$ \\ 1 Department of Sports Science and Physical Education, The Chinese University of Hong Kong, \\ Shatin, Hong Kong; damien.sutanto@link.cuhk.edu.hk (D.S.); robinho@cuhk.edu.hk (R.S.T.H.); \\ yyang@cuhk.edu.hk (Y.Y.) \\ 2 Department of Health and Physical Education, The Education University of Hong Kong, Taipo, Hong Kong; \\ ericpoon@eduhk.hk \\ * Correspondence: hsswong@cuhk.edu.hk
}

check for updates

Citation: Sutanto, D.; Ho, R.S.T.; Poon, E.T.C.; Yang, Y.; Wong, S.H.S. Effects of Different Trunk Training Methods for Chronic Low Back Pain: A Meta-Analysis. Int. J. Environ. Res. Public Health 2022, 19, 2863. https:// doi.org/10.3390/ijerph19052863

Academic Editor: Paul B. Tchounwou

Received: 20 January 2022

Accepted: 25 February 2022

Published: 1 March 2022

Publisher's Note: MDPI stays neutral with regard to jurisdictional claims in published maps and institutional affiliations.

Copyright: (c) 2022 by the authors. Licensee MDPI, Basel, Switzerland. This article is an open access article distributed under the terms and conditions of the Creative Commons Attribution (CC BY) license (https:// creativecommons.org/licenses/by/ $4.0 /)$.

\begin{abstract}
We conducted a systematic review and meta-analysis comparing motor control, isometric, and isotonic trunk training intervention for pain, disability, and re-injury risk reduction in chronic low back pain patients. The EMBASE, MEDLINE, CENTRAL, PsycINFO, SPORTDiscus, and CINAHL databases were searched from inception until 25 February 2021 for chronic low back pain intervention based on any trunk training. Outcomes include the Oswestry Disability Index (ODI) and Roland Morris Disability Questionnaire (RMDQ) for disability, the Numerical Pain Rating Scale (NPRS) for pain, and the Sorensen Test (ST) for future risk of re-injury. Isometric training was superior to the control with a mean difference $(\mathrm{MD})=-1.66,95 \%$ confidence interval $(\mathrm{CI})[-2.30,-1.01]$ in pain reduction; $\mathrm{MD}=-7.94,95 \% \mathrm{CI}[-10.29,-5.59]$ in ODI; $\mathrm{MD}=-3.21,95 \% \mathrm{CI}[-4.83,-1.60]$ in RMDQ; and $\mathrm{MD}=56.35 \mathrm{~s}, 95 \% \mathrm{CI}[51.81 \mathrm{~s}, 60.90 \mathrm{~s}]$ in ST. Motor control was superior to the control with a MD $=-2.44,95 \%$ CI [-3.10, -1.79] in NPRS; MD $=-8.32,95 \%$ CI [ - 13.43, -3.22] in ODI; and $\mathrm{MD}=-3.58,95 \% \mathrm{CI}[-5.13,-2.03]$ in RMDQ. Isometric and motor control methods can effectively reduce pain and disability, with the isometric method reducing re-injury risk.
\end{abstract}

Keywords: low back pain; rehabilitation; exercise therapy

\section{Introduction}

Most people experience low back pain within their lifetime [1,2], with mild to severe symptoms [3]. Physicians can identify the cause of pain $10 \%$ of the time, often giving a non-specific diagnosis [1]. Up to one-third of non-specific low back pain patients develop chronic symptoms persisting over three months [4], with a global prevalence of around $25 \%$ of the working-age population [4,5]. Trunk muscle training is often prescribed for the treatment of chronic low back pain (CLBP). Trunk muscle tension increases the spine's ability to remain in a neutral pose under load [6] whereas spine posture and movement away from a neutral pose under load can increase the risk of spine tissue damage [7-9]. There is considerable trunk muscle training with no explicit clinical guidelines on the types considered effective. With CLBP significantly increasing the risk of co-morbidities including musculoskeletal, neuropathic, and psychological issues [10], we need to better understand the effectiveness of different trunk muscle training methods.

Trunk training comes in many forms [11-13], and most can be classified based on the biomechanical properties and training focus as:

1. Isometric (IM) training: loading the spine while the trunk muscles contract to maintain the spine in a neutral position [14]. Plank, bird-dog, and side bridge are some isometric trunk training examples.

2. Isotonic (IT) training: moving the lumbar spine through a range of motion while under load, both eccentrically and concentrically $[13,15]$. Sit-up and back extension focused on segmental spine movement are examples of isotonic trunk training. 
3. Motor control (MC) training: isolated activation of the deep trunk musculature targeting the transverse abdominis, lumbar multifidus, diaphragm, and pelvic floor [12,16]. Some examples of this include focusing on abdominal drawing-in manoeuvre or abdominal hollowing in isolation at different positions.

Similar exercises with different training methods result in different muscle activation [17]. Altered trunk muscle activation patterns may cause CLBP, hence the MC method prioritises isolated deep activation [12]. Some types of CLBP injury can be exacerbated by a full range of motion [9] or movement under compression [8,18], hence the IM method focuses on training trunk muscle endurance while minimising spine loading [19].

Existing primary studies comparing different types of trunk training have resulted in inconsistent findings. MC could be more [20] or less effective [21] than IM in pain reduction based on the Numerical Pain Rating Scale (NPRS); more [22] or less effective [23] than IT in pain reduction; or just as [24] or more [25] effective than the control group in disability reduction based on the Roland Morris Disability Questionnaire (RMDQ). In some cases, combining different training methods also yielded inconclusive results [26,27]. Recent meta-analysis either did not specify details on the measured outcome used for disability [28], or combined the RMDQ and Oswestry Disability Index (ODI) measurements into a single outcome [27]. RMDQ and ODI have different sensitivity depending on the patient's disability level [29], and combining them may not be appropriate. There is a lack of evidence on the comparative effectiveness of different trunk training methods in pain and disability reduction, along with the future risk of CLBP re-injury.

This study aimed to evaluate and synthesise the comparative effectiveness of MC, IM, and IT trunk training methods using a meta-analysis based on validated outcomes. This novel approach would provide clinical practitioners with more specific guidelines on CLBP training prescription. Subgroup analysis was performed to compare the effect of training duration and patient age on different trunk training methods. The subgroup analysis can provide more insights on how training duration and patient age can affect trunk training effectiveness.

\section{Materials and Methods}

\subsection{Experimental Approach to the Problem}

This study was in accordance with the Preferred Reporting Items for Systematic Reviews and Meta-Analyses (PRISMA) guidelines on systematic reviews and meta-analyses [30]. The protocol was registered on the International Prospective Register of Systematic Reviews (PROSPERO; Registration No. CRD42020168972).

Electronic databases (EMBASE, MEDLINE, PsycINFO, CENTRAL, CINAHL, and SPORTDiscus) were searched from inception until 25 February 2021, and peer-reviewed. No language restriction was applied, with a complete search strategy based on a past Cochrane review [31] available in Appendix A. Non-English journal articles were translated into English using Google Translate before data extraction. Data extracted from each study included subject demographics, inclusion and exclusion criteria, intervention details, and outcome measures.

\subsection{Subjects}

This study focused on the working-age population (19-60 years) as a significant percentage of this population experience CLBP [5,32]. The 19-25 year old population group may have different causes of CLBP and respond differently to training treatment compared to the 50-60 year old population group. Due to the non-specific nature of the CLBP diagnosis given to the 19-60 year population group, recent meta-analyses on CLBP intervention [26-28] have focused on this population as a whole. Many intervention studies have either used a similar age range [21,23] or do not specify any age range [33,34] in their subject recruitment criteria. CLBP is defined as persistent low back pain for at least 12 weeks [3,35] or as diagnosed by a clinician. Studies on patients with osteoarthritis, cancer, or cardiovascular disease such as claudication were excluded as their underlying 
condition may cause CLBP [36-38]. Randomised controlled trials (RCT) with patients that had undergone major spinal surgery were excluded as surgery may change the muscle, fascia, or neural structure around the lumbar area $[39,40]$.

\subsection{Procedures}

Interventions compared for this study were trunk training that could be classified as IM, IT, or MC. Training that could not be exclusively classified as either was excluded [41,42]. Interventions combining trunk training with aerobic or limb strength training exceeding 15 min were excluded as aerobics and limb strength training may reduce the pain and disability of CLBP patients [28,43].

The included RCTs had to contain either a control or a different trunk training group as a comparator in the pre- and post-intervention. The control included passive interventions considered ineffective for CLBP, placebo intervention, or simple advice such as maintaining active daily living or exercise avoidance. Passive interventions included transcutaneous electrical nerve stimulation (TENS) [44], ultrasound [45], and patient education only [46]. Placebo intervention included detuned ultrasound, TENS, and sham massage. Home exercise prescriptions were not considered as a valid control because patient training adherence may differ and cause high measurement variability. Flexibility and mobility are not significant predictors of future CLBP [47] and have no significant effect on pain and disability reduction [28], hence brief warm-up stretching and mobility exercises on the intervention or control were acceptable.

Outcomes can be classified as Patient-Reported Outcome Measures (PROM) or Patient Performance Test (PPT) based on the COSMIN guidelines for CLBP. A recent systematic review concluded that ST has high test-retest, intra-rater, and inter-rater reliability for PPT [48]. ST is inversely correlated to CLBP risk across the study population of interest $[49,50]$ and was chosen as the PPT for the meta-analysis.

PROMs are outcomes based on the participant's subjective responses. A recent Delphi study concluded that NPRS, ODI, and RMDQ are the most widely accepted PROMs for CLBP intervention [51]. This review only included clinical intervention studies excluding cohort studies, case studies, commentaries, and editorials. Patients scoring $61 \%$ or above on the ODI were considered to have a crippling disability [52] and should be receiving positive intervention instead of physical training [29]. Hence, studies with patients from this group were excluded from the analysis.

Current international guidelines for the treatment of CLBP do not recommend CLBP patient subgrouping $[3,35,53,54]$. RCTs that use specific classification to separate patients into different treatments were excluded to ensure the external validity of the meta-analysis. Article titles and abstracts identified from the search results were independently assessed by two reviewers, with the primary research data exported to Endnote X9.2 build 13,018. Relevant grey literature was searched for related trial data. Full-text articles were screened independently by both reviewers, with a third reviewer adjudicating any disagreement. Published articles with the most relevant outcomes were included in the analysis for multiple publications from a single RCT. Publication data (author, year, and origin), study design (patients and groups numbers), intervention, and outcome from included RCTs were extracted to Table A1 in Appendix B, with the primary authors being contacted for missing data.

Review Manager v5.4.1 (Cochrane Collaboration, Copenhagen, Denmark) was used for the statistical analyses. Before input in the meta-analysis, pre- and post-intervention mean differences (MD) and standard deviations (SD) from the included studies were converted to change MD and SD based on Cochrane handbook section 16.1.3.2. Significance was set for $\alpha$ at $5 \%$ and $95 \%$ confidence interval (CI), and all analyses used the random effect model. Pain VAS reported as a score of 0-100 was standardised to NPRS 0-10. Weight column indicated in the meta-analysis result in Review Manager indicates the effect percentage from a particular study towards the overall MD. Green squares on meta-analysis result graphically indicates the effect of each individual study while black squares indicate the 
overall effect of the combined studies. Heterogeneity as I2 was classified as low ( 25\%), moderate $(\sim 50 \%)$, and high $(\sim 75 \%)$ [55]. High heterogeneity could be due to publication bias, methodological issues, or clinical differences. Methodological issues were investigated under the risk of bias assessment. The clinical difference was investigated using subgroup analysis. Detailed significant and insignificant results are displayed in separate figures.

The Cochrane Risk of Bias 2.0 tool was used to assess the randomisation, assessment, missing outcome, measurement outcome, and reporting outcome bias [56]. Two researchers independently evaluated and resolved differences through discussion. Sensitivity analysis was conducted by removing the high risk of bias studies. Result certainty was assessed based on the result and heterogeneity change after sensitivity analysis.

The trunk training clinical trial durations ranged from one [33] to twelve months [57]. Training duration subgroup analysis could justify a longer duration training prescription for severely affected CLBP patients. Patients under 40 may have a 3.7 times higher chance of pain and disability reduction than older patients [58]. Some intervention studies recruited patients with an age range that overlapped 40 [21,34]. Patient mean age was used to group the included studies. Studies were grouped into under-40, 40-45, and over-45 years old, with the analysis only comparing the under-40 and over 45-groups. Effects of ageing on human physiology and related training adaptation is gradual and non-uniform. Some studies with participants with a mean age of 40-45 may have an overall physiology closer to those under 40 , while in other studies, the overall physiology was closer to those over 45. The 40-45 age group was not analysed to remove uncertainty in their classification as more similar to those under- 40 or above- 45 in the subgroup analysis. Intervention studies with the participants' mean age of different group belonging to different classification were excluded. This subgroup analysis provided an insight into the effect of patient age on CLBP training effectiveness.

\section{Results}

The literature search identified 10,846 citations with 10,372 citations excluded after screening the title and abstracts and full-text screening of the remaining 476 citations. One study with a related intervention and outcome was excluded as all the subjects later received surgical intervention prior to post-intervention outcome measurement [59]. Studies with non-chronic low back pain subjects were excluded $[60,61]$ as the majority of low back pain cases resolve spontaneously within 6 weeks [62]. Forty-seven RCTs $(\mathrm{N}=2299)$ were included in the meta-analysis. A PRISMA flowchart [30] of the RCT search and selection results is shown in Figure 1.

Individual study characteristics of the included RCTs are mentioned in Table A1 in Appendix B. Included RCTs were from 19 countries: 26 studies from Asia, 15 from Europe, five from America, and one from Australia. One study was in the Korean language only, while the remaining 44 were in English and another language or were written only in English. In studies with a control, seven had no details on the control intervention, five received exercise avoidance advice, four received patient education, four received passive treatment, three received a passive placebo treatment, and three were put on a waiting list. The number of patients per intervention group at baseline ranged from 5-84, with 22 of the 47 RCTs having 20-40 patients per group. Six of the included RCTs had over 40 patients per group. 


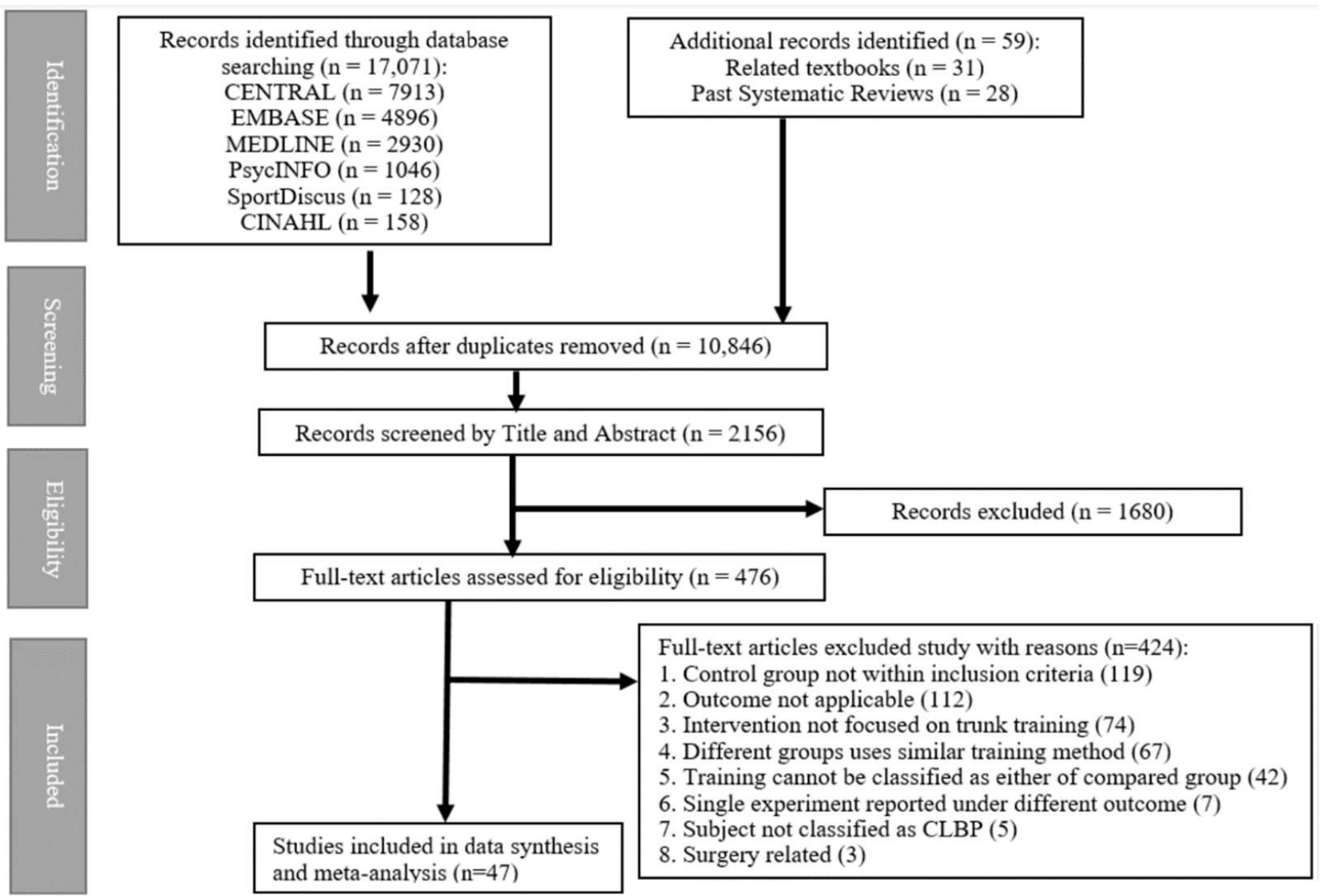

Figure 1. Flowchart of literature selection in the systematic reviews on trunk muscle training for chronic low back pain.

Two studies recruited male patients only. Five studies did not specify their patient gender demographics. Ten studies exclusively recruited female patients and 30 studies recruited both male and female patients. Nine of the studies used four weeks as the duration for training intervention, 15 used six weeks, 14 used eight weeks, two used 10 weeks, and seven used 12 weeks. Sixteen studies used patients with a mean age below 40 , nine studies used patients with a mean age $40-45$, and 12 studies used patients with a mean age above 45 years old. Seven of the included studies had intervention arms belonging to different age groups and three did not have information on their recruited subject age.

IM intervention was more effective than the control in reducing pain as measured by NPRS (Figure 2, first row), MD $=-1.66,95 \%$ CI [ $-2.30,-1.01]$, I2 $=90 \%, p<0.01$. IM was superior to the control in disability reduction as measured by ODI (Figure 2, second row), $\mathrm{MD}=-7.94,95 \% \mathrm{CI}[-10.29,-5.59], \mathrm{I} 2=60 \%, p<0.01$, and RMDQ (Figure 2, third row), $\mathrm{MD}=-3.21,95 \% \mathrm{CI}[-4.83,-1.60], \mathrm{I} 2=84 \%, p<0.01$. IM intervention increased trunk extensor endurance (Figure 2,17th row) compared to that of the control with $\mathrm{MD}=56.35$, $95 \%$ CI $[51.81,60.90]$, I $2=0 \%, p<0.01$. 
Numerical Pain Rating Scale Outcome

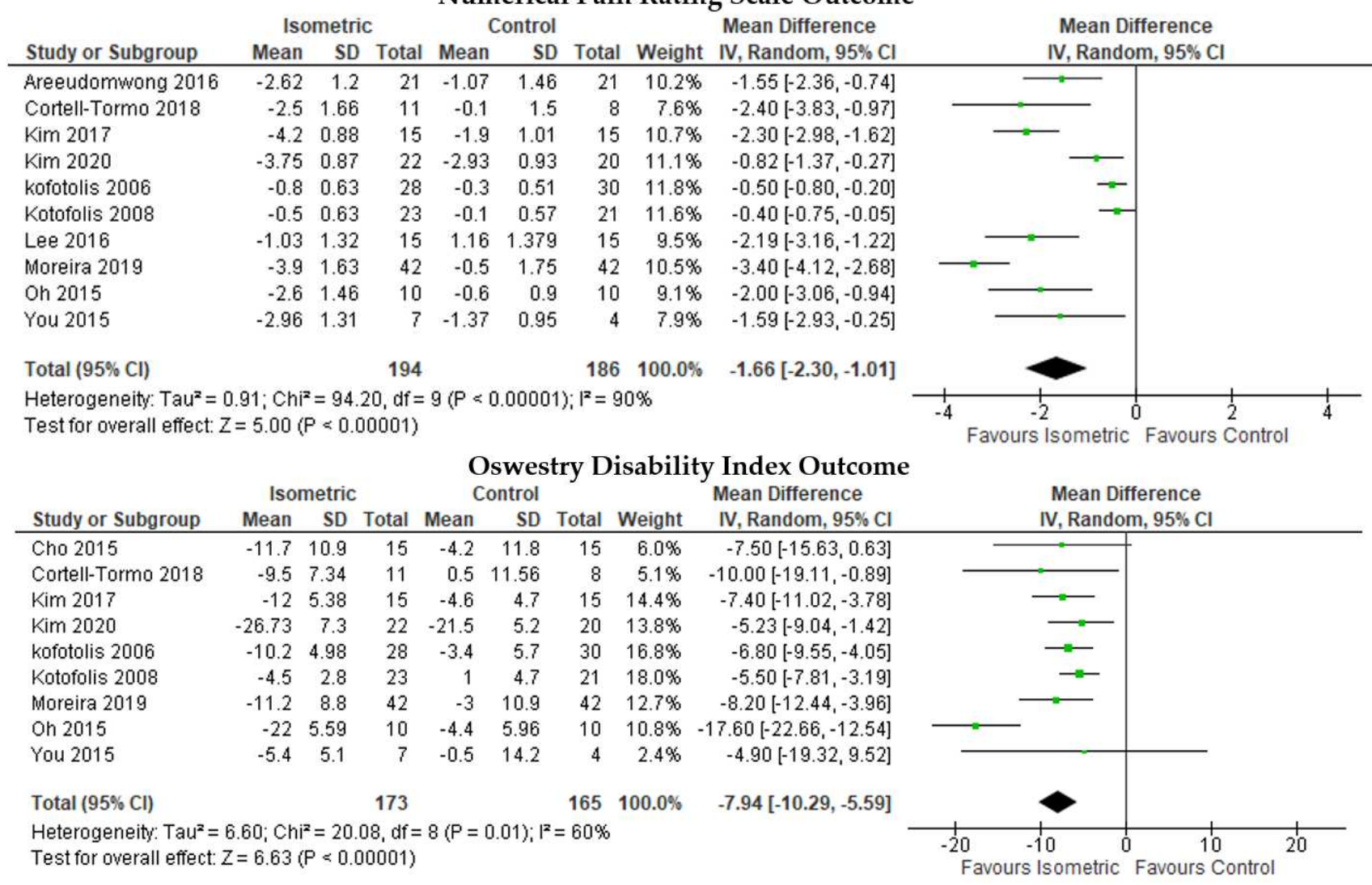

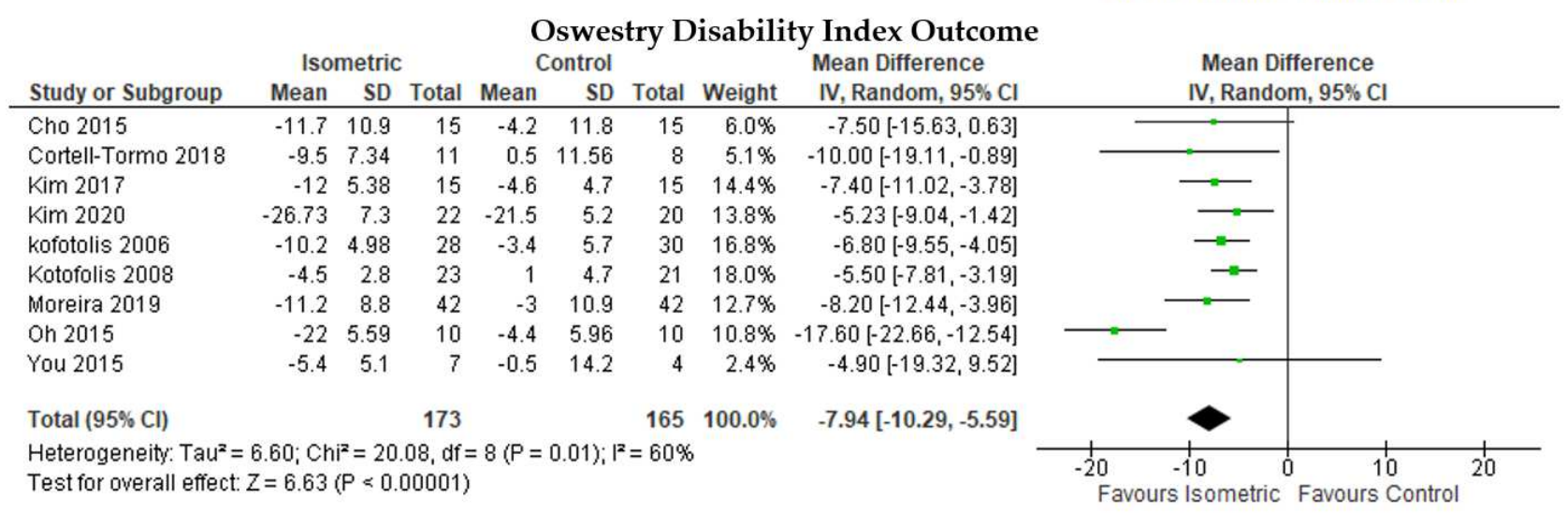

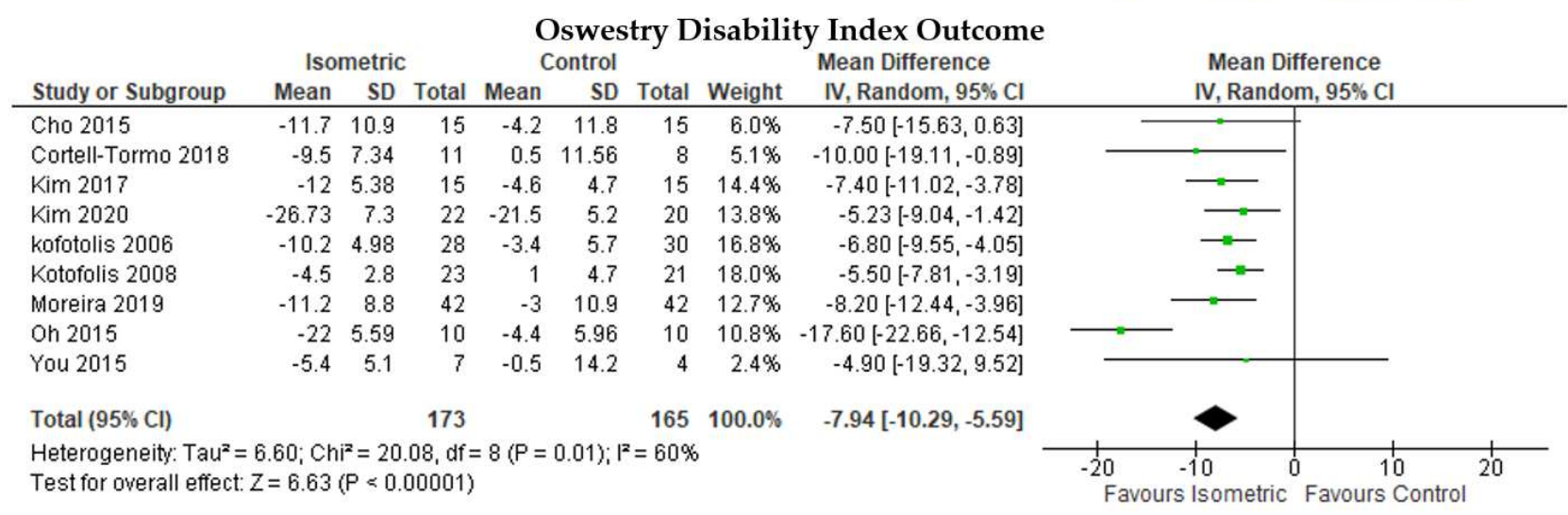

Figure 2. Pairwise meta-analyses on the effectiveness of isometric trunk muscle training compared to the control for chronic low back pain. 
MC was more effective in reducing pain than the control as measured by the NPRS (Figure 2, first row), $\mathrm{MD}=-2.44,95 \%$ CI $[-3.10,-1.79], \mathrm{I} 2=79 \%, p<0.01$. MC was superior in controlling disability reduction as measured by the ODI (Figure 3, second row), $\mathrm{MD}=-8.32,95 \% \mathrm{CI}[-13.43,-3.22], \mathrm{I} 2=43 \%, p<0.01$ and as measured by RMDQ (Figure 3, third row), $\mathrm{MD}=-3.58,95 \% \mathrm{CI}[-5.13,-2.03], \mathrm{I} 2=47 \%, p<0.01$.

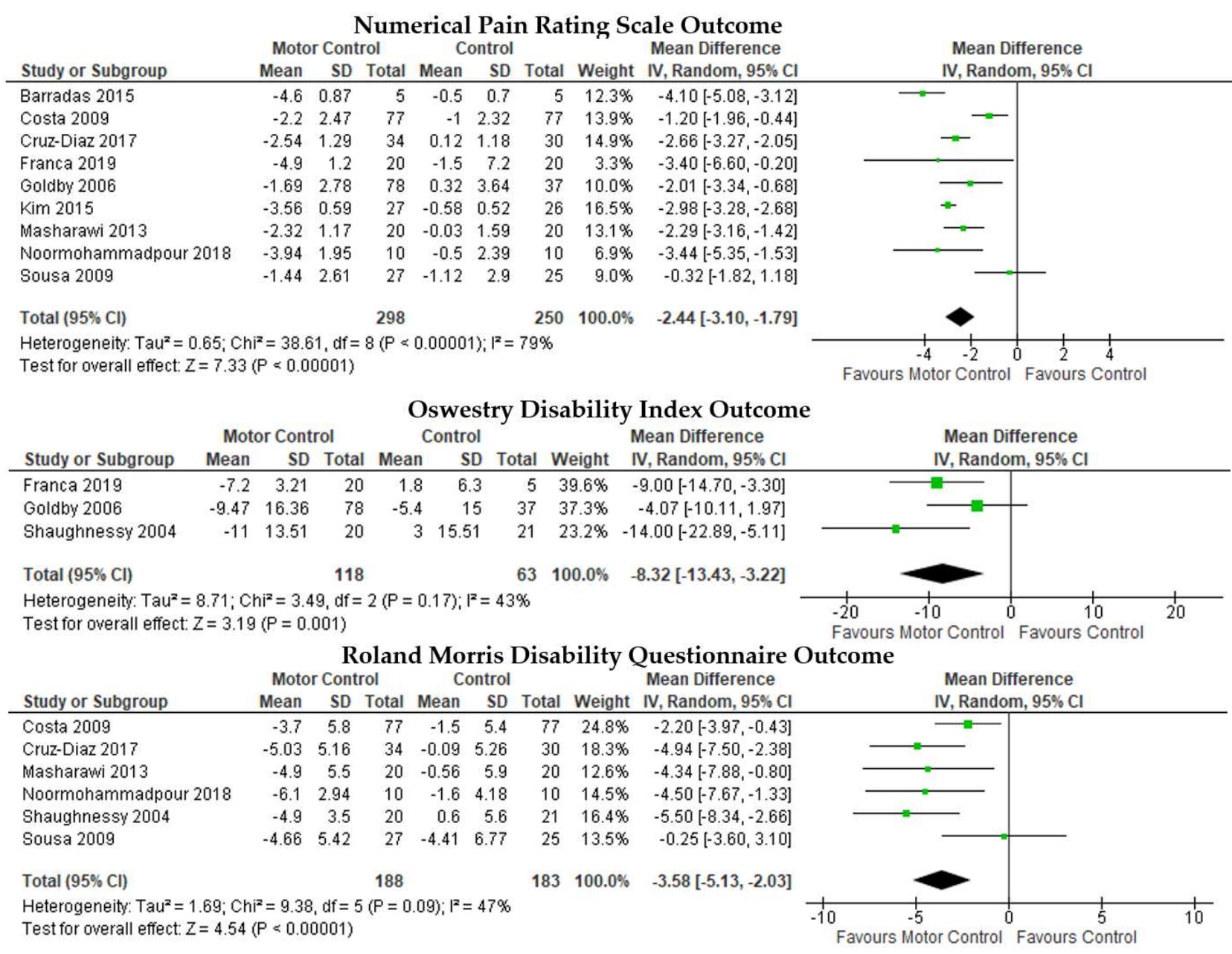

Figure 3. Pairwise meta-analyses on the effectiveness of motor control trunk muscle training compared to the control for chronic low back pain.

A pairwise meta-analysis comparing different training methods resulted in $\mathrm{MC}$ being superior to IT on the NPRS (Figure 4, first row), MD $=-0.84,95 \%$ CI $[-1.56$ to -0.11$]$, $\mathrm{I} 2=86 \%, p=0.02$, and ODI (Figure 4, second row), MD $=-4.66,95 \%$ CI [ $-7.67,-1.65]$, I2 $=84 \%, p<0.01$. MC was superior to IM in disability reduction based on the ODI (Figure 4 , third row), $\mathrm{MD}=-5.95,95 \% \mathrm{CI}[-10.77,-1.12], \mathrm{I} 2=88 \%, p=0.02$. The difference was not significant as measured with the NPRS (Figure 4, fourth row), MD $=-0.09,95 \%$ CI $[-0.42$, $0.24], \mathrm{I} 2=67 \%, p=0.61$, and RMDQ (Figure 4, fifth row), MD = 0.78, 95\% CI [-0.66, 2.22], $\mathrm{I} 2=22 \%, p=0.29$. 


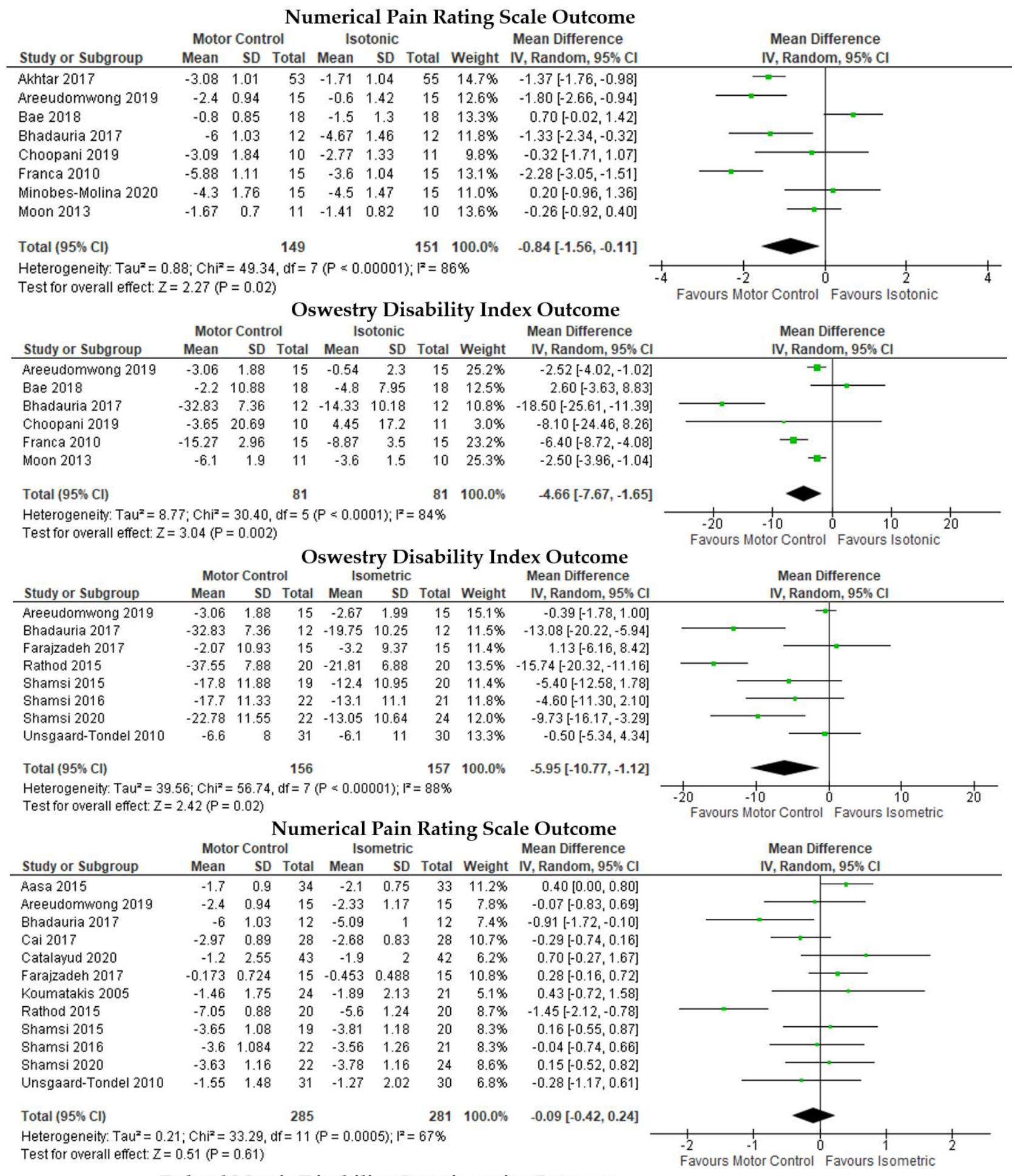

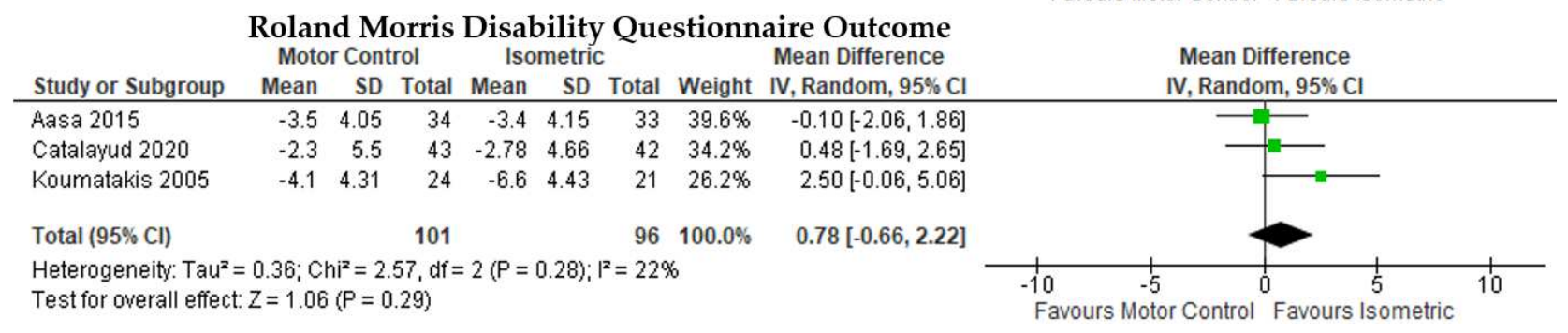

Figure 4. Pairwise meta-analyses on the comparative effectiveness of different trunk muscle training methods for chronic low back pain. 
IT intervention did not result in significant NPRS reduction compared to the control (Figure 5, first row), with MD $=-0.87,95 \%$ CI $[-2.05,0.31]$, I2 $=74 \%, p=0.15$, while IM and IT intervention were not significantly different in the NPRS (Figure 5, second row) with $\mathrm{MD}=0.19,95 \% \mathrm{CI}[-0.36,0.74], \mathrm{I} 2=65 \%, p=0.50$. IT significantly reduced disability as measured with ODI compared to the control (Figure 5, third row) with MD $=-11.22$, $95 \%$ CI $[-18.01,-4.42], \mathrm{I} 2=77 \%, p=0.001$. In addition, IT was not significantly different to IM in ODI reduction (Figure 5, fourth row) with MD $=0.25,95 \%$ CI $[-2.24,2.74]$, $\mathrm{I} 2=74 \%, p=0.85$. MC to IT comparison in the RMDQ outcome did not show any significant difference (Figure 5, fifth row), with $\mathrm{MD}=0.42,95 \% \mathrm{CI}[-0.83,1.67], \mathrm{I} 2=0 \%, p=0.51$. The IT to control comparison resulted in the largest disability (ODI) reduction $(\mathrm{MD}=-11.22)$ while $\mathrm{MC}$ was more effective than IT $(\mathrm{MD}=-4.66)$.

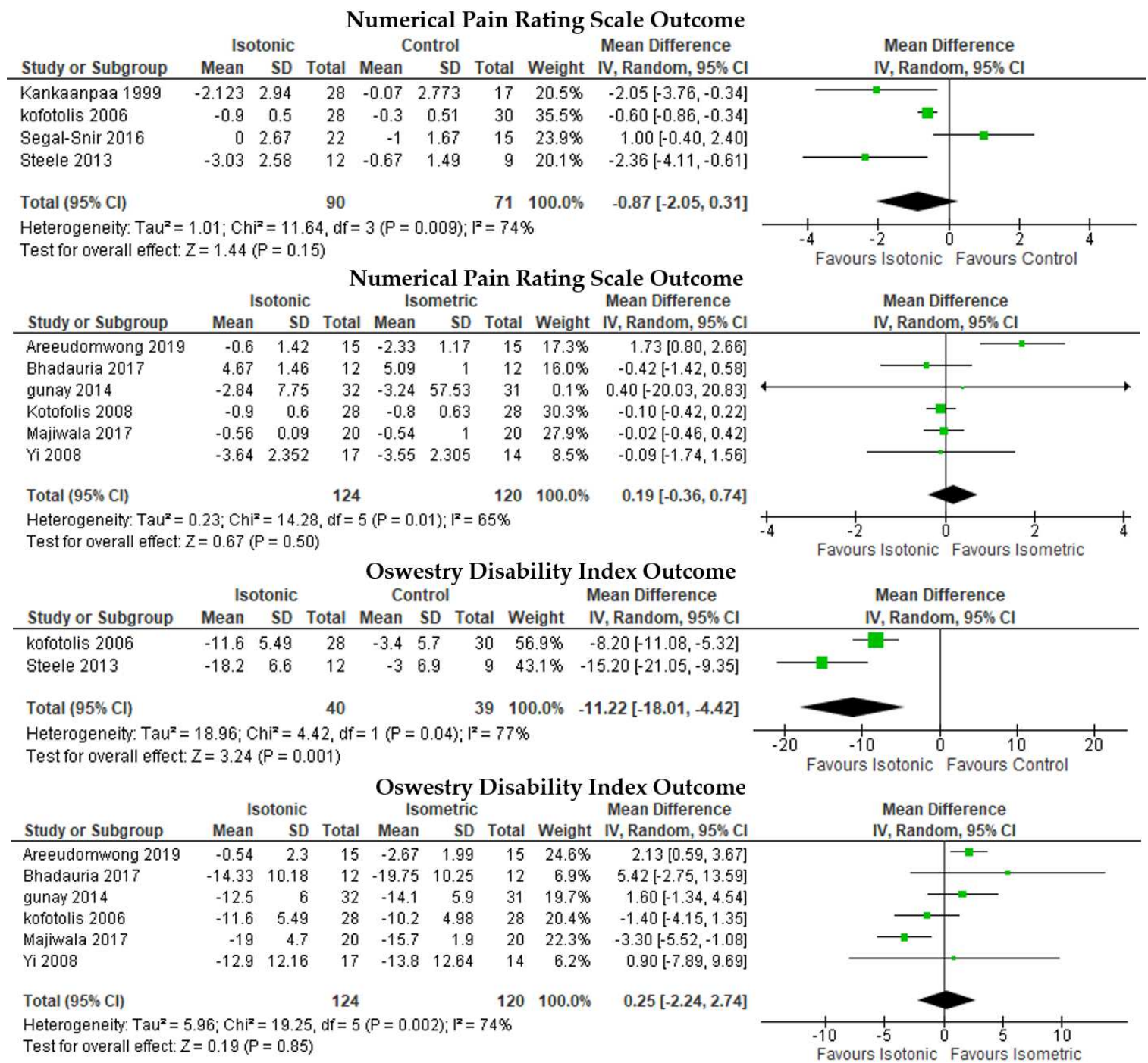

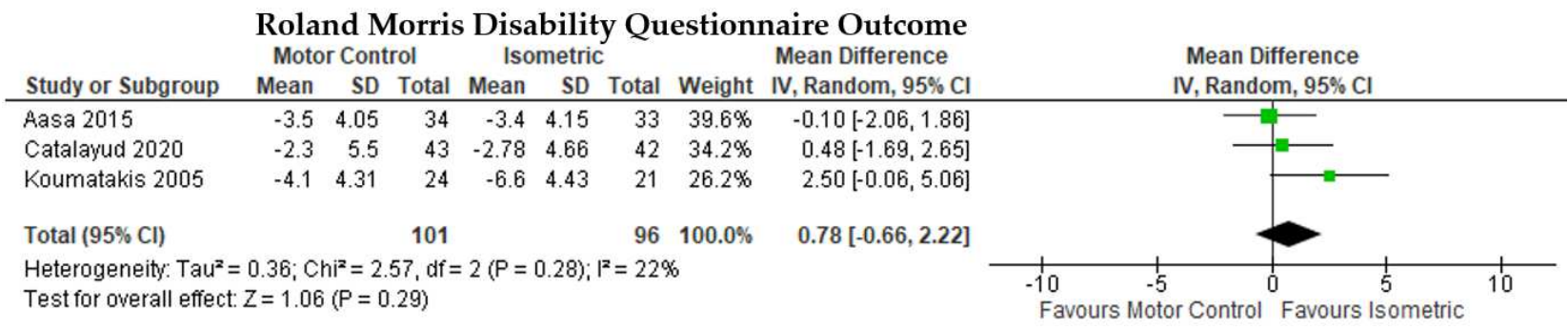

Figure 5. Pairwise meta-analyses with no significant results. 


\subsection{Sensitivity Analysis}

Figure 6 lists the included studies along with the risk of bias in the five bias domains. Studies with low risk of bias in all five domains were judged as having low overall risk of bias. Studies with concerns in their methodology in two or less domains were judged as having some concerns in the overall risk of bias. Studies with three or more domains having methodological concerns or with high risk of bias in one of the domains were judged as having high risk in overall risk of bias. Overall, four studies had a low risk of bias, 21 had some concerns, and 21 had a high risk of bias. Ninety percent of the concerns in the randomisation bias were due to a lack of allocation concealment in the study report. Over $60 \%$ of the studies had some concerns on measurement outcome bias due to the lack of assessor blinding on group allocation, with PROMs being subjective in nature. The 21 studies with a high risk of bias were removed in the sensitivity analysis, while another study [63] was removed from the NPRS results due to missing data.

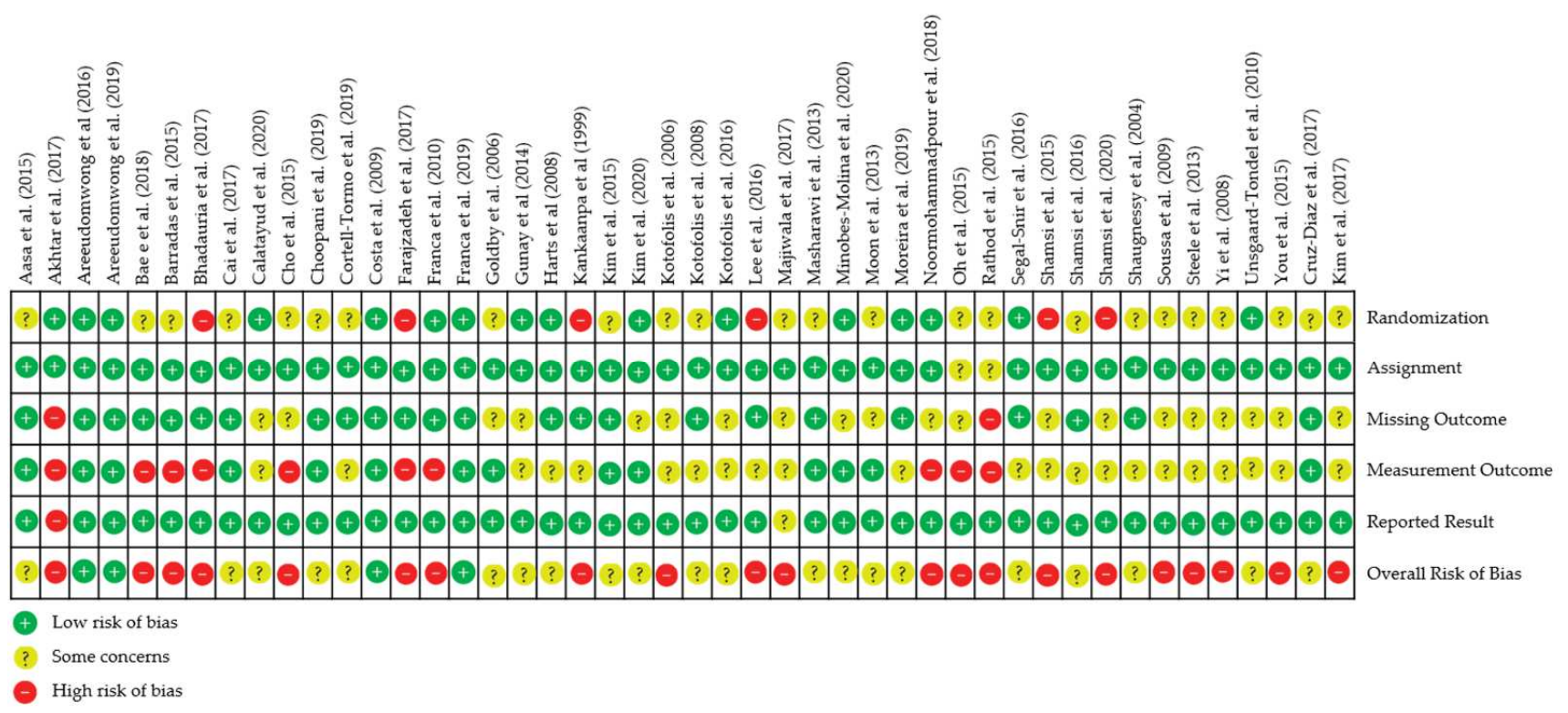

Figure 6. Risk of bias among the included randomised controlled trials.

Difference in MC and IM effect on disability reduction as measured by the ODI became non-significant (Appendix C.1, sixth row), $\mathrm{MD}=-0.56,95 \% \mathrm{CI}[-1.86,0.75]$, $\mathrm{I} 2=0 \%, p=0.40$. The difference remained non-significant as measured with the NPRS (Appendix C.1, first row), $\mathrm{MD}=0.08,95 \% \mathrm{CI}[-0.21,0.37], \mathrm{I} 2=24 \%, p=0.58$. IM remained more effective than the control based on the NPRS (Appendix C.1, second row), $\mathrm{MD}=-1.55,95 \% \mathrm{CI}[-2.28,-0.83], \mathrm{I} 2=92 \%, p<0.01$, and ODI (Appendix C.1, seventh row), $\mathrm{MD}=-6.43,95 \% \mathrm{CI}[-7.80,-5.07] ; \mathrm{I} 2=0 \%, p<0.01$. MC remained more effective than the control in pain reduction as measured by the NPRS (Appendix C.1, third row), $\mathrm{MD}=-2.12,95 \% \mathrm{CI}[-2.89,-1.35], \mathrm{I} 2=83 \%, p<0.01$. In addition, $\mathrm{MC}$ remained more effective in disability reduction as measured by the ODI (Appendix C.1, eighth row), $\mathrm{MD}=-10.46,95 \% \mathrm{CI}[-15.25,-5.66], \mathrm{I} 2=0 \%, p<0.01$, and RMDQ (Appendix C.1, eleventh row), $\mathrm{MD}=-3.44,95 \% \mathrm{CI}[-5.24,-1.63], \mathrm{I} 2=55 \%, p<0.01$. MC was no longer superior to IT in NPRS reduction (Appendix C.1, fourth row) with MD $=-0.59,95 \%$ CI $[-1.49,0.32], \mathrm{I} 2=71 \%, p=0.20$, while remaining superior in ODI reduction (Appendix C.1, ninth row), with $\mathrm{MD}=-2.53,95 \% \mathrm{CI}[-3.58,-1.49] ; \mathrm{I} 2=0 \%, p=0.01$.

Regarding IM to IT comparison in the NPRS outcome, four of the six included studies were removed due to a high risk of bias with a sensitivity analysis showing no significant difference (Appendix C.1, fifth row), MD =0.76, 95\% CI [-1.03, 2.55], I2 =92\%, $p=0.40$. In the IM to IT comparison in the ODI outcome, three of the six included studies had a high 
risk of bias according to risk of bias analysis, with no significant difference in sensitivity analysis (Appendix C.1, tenth row), MD =0.76, 95\% CI $[-1.03,2.55], \mathrm{I} 2=92 \%, p=0.40$.

\subsection{Training Duration Subgroup Analysis}

The difference between IM and MC effects on the NPRS (Appendix C.2, first row) outcome was insignificant for under eight weeks of intervention, $\mathrm{MD}=-0.25,95 \% \mathrm{CI}$ $[-0.74,0.24], \mathrm{I} 2=74 \%, p=0.32$, and for eight or more weeks of intervention, $\mathrm{MD}=0.14$, $95 \%$ CI $[-0.28,0.55], \mathrm{I} 2=47 \%, p=0.52$. Compared to a total heterogeneity of $67 \%$, subgroup analysis resulted in lower heterogeneity in long-term intervention and higher heterogeneity in short-term intervention with no significant difference between the two groups, $p=0.24$.

IM comparison with the control in the NPRS outcome (Appendix C.2, second row) was significant for under eight weeks, $\mathrm{MD}=-1.10,95 \% \mathrm{CI}[-1.65,-0.54], \mathrm{I} 2=84 \%$, $p<0.01$ and for eight or more weeks of training duration, $\mathrm{MD}=-2.58,95 \% \mathrm{CI}[-3.32$, -1.83 ], I $2=54 \%, p<0.01$. Compared to the total heterogeneity of $90 \%$, subgroup analysis resulted in lower heterogeneity in both subgroups and greater pain reduction in longer duration interventions $(p<0.01)$.

MC comparison with the control (Appendix C.2, third row) resulted in significant NPRS reduction for under eight weeks of intervention, $\mathrm{MD}=-2.51,95 \% \mathrm{CI}[-4.12,-0.89]$, I $2=91 \%, p<0.01$ and for eight or more weeks of intervention, MD $=-2.47,95 \% \mathrm{CI}[-3.15$, -1.79 ], I $2=64 \%, p<0.01$. Compared to a total heterogeneity of $82 \%$, subgroup analysis resulted in lower heterogeneity in long-term intervention and higher heterogeneity in short-term intervention with no significant difference between the two groups $(p=0.86)$.

IM comparison with the control in ODI outcome (Appendix C.2, fourth row) was significant for a training duration of under eight weeks, $\mathrm{MD}=-6.17,95 \% \mathrm{CI}[-7.61$, $-4.74], \mathrm{I} 2=0 \%, p<0.01$ and for eight or more weeks, MD $=-12.07,95 \% \mathrm{CI}[-18.72,-5.41]$, $\mathrm{I} 2=75 \%, p<0.01$. Compared to a total heterogeneity of $60 \%$, subgroup analysis resulted in lower heterogeneity in shorter duration while increasing heterogeneity in longer duration, with no significant difference between the two groups $(p=0.09)$.

\subsection{Age Subgroup Analysis}

Subgroup analysis on IM intervention indicated that IM was effective in pain (NPRS) reduction in all age groups (Appendix C.3, first row). Patients under 40 years experienced greater pain reduction, $\mathrm{MD}=-1.99,95 \% \mathrm{CI}[-2.44,-1.53], \mathrm{I} 2=0 \%, p<0.01$, compared to patients over 45 years of age, $\mathrm{MD}=-1.32,95 \% \mathrm{CI}[-2.46,-0.18], \mathrm{I} 2=73 \%, p=0.02$. Heterogeneity of both groups was lower than the total heterogeneity of $\mathrm{I} 2=84 \%$ with a significant difference within the groups $(p=0.01)$. IM intervention was also effective in disability reduction (ODI) among all age groups (Appendix C.3, third row). Patients under 40 years experienced similar disability reduction, $\mathrm{MD}=-7.61,95 \% \mathrm{CI}[-10.88,-4.33]$, $\mathrm{I} 2=0 \%, p<0.01$, compared with that of patients over 45 years of age, MD $=-10.16,95 \%$ CI $[-18.66,-1.66], \mathrm{I} 2=87 \%, p=0.02$. The heterogeneity of the under 40 group was lower, $\mathrm{I} 2=0 \%$, than the total heterogeneity of I $2=65 \%$ with the highest heterogeneity in the over 45 group, I2 $=87 \%$. There was no significant difference within the groups $(p=0.49)$.

Subgroup analysis based on age indicated that MC intervention was effective in pain reduction (NPRS) in all age groups (Appendix C.3, second row). Patients under 40 years experienced significantly greater pain reduction, $\mathrm{MD}=-3.11,95 \% \mathrm{CI}[-3.70,-2.52]$, $\mathrm{I} 2=67 \%, p<0.01$, compared to that in patients over 45 years of age, $\mathrm{MD}=-1.39,95 \% \mathrm{CI}$ $[-2.40,-0.39], \mathrm{I} 2=68 \%, p<0.01$. Heterogeneity of both groups were lower than the total heterogeneity of I $2=79 \%$, with a significant difference between the subgroups $(p=0.02)$.

\section{Discussion}

Both IM and MC interventions resulted in clinically significant pain and disability reduction in CLBP patients according to the ACP definition [32]. IM methods may also be effective in CLBP re-injury risk reduction based on increased trunk extensor endurance [47]. All three intervention groups have often been grouped as one in past syntheses [27,28], 
which resulted in lower pain or disability reduction as IT intervention was not effective in pain (NPRS) and disability (RMDQ) reduction. Sensitivity analysis resulted in IM and MC interventions being effective in pain and disability reduction. IT was ineffective in reducing pain (NPRS), possibly due to the training loading that imitates some patient-specific spine injury mechanisms [7-9,18].

Past intervention studies have indicated that single postural re-education intervention can reduce pain and disability in acute and chronic low back pain patient $[64,65]$. This result is consistent with the recent meta-analysis on postural re-education for CLBP [66]. MC and IM may be equally effective as both focus on developing the muscular endurance to hold the spine in a neutral position including during limb movement progressions that may have a similar effect with global postural re-education. Most included RCTs comparing both interventions equalised training intensity by having an IM group training duration $30-50 \%$ less than that of the MC group, which may cause both groups to have similar outcomes [67-70].

Inconsistent results in pair-wise meta-analyses with the IT method could be due to the small number of included studies within some pairwise meta-analyses, high risk of bias in some of the included studies, no standard in trunk training frequency and duration, and variability in the recruited patient age group and training duration.

Only three of the included studies used ST in comparing MC and IM [21,34] and only two included studies comparing IT to IM [63,71]. Standardisation and use of a select set of objective outcomes would provide better comparison in future meta-analyses.

Subgroup analysis on CLBP patients trained with IM methods indicated that a longer training duration resulted in further pain reduction, while disability reduction was not significantly different. This indicates that IM intervention may reduce disability earlier than pain. The training duration did not significantly affect pain reduction in MC intervention, indicating that other factors such as clinician skill or difference in training prescription may have an impact that is more significant. Age subgroup analysis indicated that both MC and IM intervention was effective in all age groups, with patients under 40 years experiencing greater pain reduction compared to those over 45 years of age. This could be because older patients require a higher training stimulus to achieve comparable muscular adaptation as that in younger patients [72].

Limitations of the current meta-analysis include a lack of analysis on gender difference, effects of training intensity, and comparison between isolated trunk training and progression with limb movement due to insufficient data. The effect of patient grouping based on specific assessment exceeded the scope of this study. Future research could focus on a single training method in one intervention group to enable a better understanding of the effects of a specific trunk training method on CLBP patient outcomes. In addition, future RCTs should consider incorporating the NPRS, ODI, RMDQ, and ST measurement and follow the CONSORT [73] guidelines to reduce the risk of bias and increase methodological transparency. Future meta-analyses should consider the difference in the recruited patient age demographic and training duration when comparing the different types of CLBP interventions. Other outcome measures and multi-modal interventions may be useful, however, these were excluded to limit the scope of this study.

\section{Conclusions}

Clinicians can prescribe trunk muscle training, focusing on deep abdominal muscle activation (MC method) such as the abdominal draw-in-manoeuvre or isometric trunk muscle activation (IM method) such as the plank for patients with CLBP. Both training approaches can be effective as both methods train the trunk muscle endurance to hold the spine in a neutral position including during active daily living. As the spine in a neutral position is more resilient to tissue injury, CLBP patients trained in the MC and IM methods could gradually experience pain and disability reduction. Trunk muscle training focusing on spine movement (IT method) such as sit-ups may be less effective in pain reduction as it does not train CLBP patients to hold their spine in a neutral position. 
Short-term IM training intervention from four to six weeks can result in a pain and disability reduction. CLBP patients with a larger pain score can experience a larger pain reduction with a longer IM intervention of at least eight weeks. Both IM and MC methods may result in larger pain reduction in patients under 40 compared to those over 45 . Future CLBP intervention studies should use participants with the same mean age on different groups while future meta-analysis should consider limiting the age range of the included studies' populations. Further study on the effect of ageing on CLBP training adaptation, and how to adapt training prescription according to CLBP patient age can be investigated in future studies.

Author Contributions: Conceptualisation, D.S. and R.S.T.H.; Methodology, R.S.T.H.; Software, R.S.T.H.; Search strategy, D.S. and R.S.T.H.; Database Search and Screening D.S., R.S.T.H. and E.T.C.P.; Data synthesis, D.S. and R.S.T.H.; Writing-original draft preparation, D.S.; Writing-review and editing, R.S.T.H., E.T.C.P., Y.Y. and S.H.S.W.; Supervision, S.H.S.W. All authors have read and agreed to the published version of the manuscript.

Funding: This research received no external funding.

Institutional Review Board Statement: Not applicable.

Informed Consent Statement: Not applicable.

Data Availability Statement: The data in this study can be provided upon reasonable request to the corresponding author.

Conflicts of Interest: The authors declare no conflict of interest.

\section{Appendix A. Database Search Strategies}

CENTRAL search strategy on 24 February 2021

ID Search Statement

\#1 MeSH descriptor: [Back Pain] explode all trees

\#2 dorsalgia

\#3 backache

\#4 (lumb* or back) next pain

\#5 coccyx or coccydynia or spondylosis

\#6 MeSH descriptor: [Spine] explode all trees

\#7 MeSH descriptor: [Spinal Diseases] explode all trees

\#8 lumbago or discitis

\#9 disc near herniat*

\#10 disk NEAR herniat*

\#11 spinal fusion

\#12 facet near joint*

\#13 MeSH descriptor: [Intervertebral Disc] explode all trees

\#14 postlaminectomy

\#15 arachnoiditis

\#16 failed near back

\#17 MeSH descriptor: [Cauda Equina] explode all trees

\#18 lumb* near vertebra*

\#19 spinal near stenosis

\#20 slipped near disc ${ }^{*}$

\#21 slipped NEAR disk $^{*}$

\#22 degenerat* near disc ${ }^{*}$

\#23 degenerat* near disk ${ }^{*}$

\#24 stenosis near spine

\#25 stenosis near root

\#26 stenosis near spinal

\#27 displace* near disc ${ }^{*}$

\#28 displace* near disk*

\#29 prolap* near disc*

\#30 prolap* near disk* 
\#31 MeSH descriptor: [Sciatic Neuropathy] explode all trees

\#32 sciatic $^{*}$

\#33 back disorder*

\#1 OR \#2 OR \#3 OR \#4 OR \#5 OR \#6 OR \#7 OR \#8 OR \#9 OR \#10 OR \#11 OR \#12 OR

\#34 \#13 OR \#14 OR \#15 OR \#16 OR \#17 OR \#18 OR \#19 OR \#20 OR \#21 OR \#22 OR \#23 OR \#24 OR \#25 OR \#26 OR \#27 OR \#28 OR \#29 OR \#30 OR \#31 OR \#32 OR \#33

\#35 training

\#36 endurance

\#37 trunk stabil*

\#38 lumbar stabil*

\#39 exercise

\#40 rehab*

\#41 core stabil*

\#42 transverse abdominis

\#43 multifidus

\#44 longissimus

\#45 extensor

\#46 \#35 OR \#36 OR \#37 OR \#38 OR \#39 OR \#40 OR \#41 OR \#42 OR \#43 OR \#44 OR \#45

\#47 \#34 AND \#46

"trials" tab, 7913 results

MEDLINE search on 25 February 2021

ID Search Statement

\#1 clinical trial.mp.

\#2 clinical trial.pt.

\#3 random:.mp.

\#4 1 or 2 or 3

\#5 training*.mp.

\#6 rehab*.mp.

\#7 exercise*.mp.

\#8 lumbar stabil*.mp.

\#9 trunk stabil*.mp.

\#10 core stabil ${ }^{*}$.mp.

\#11 transverse abdominis.mp.

\#12 multifidus.mp.

\#13 longissimus.mp.

\#14 obliques.mp.

\#15 5 or 6 or 7 or 8 or 9 or 10 or 11 or 12 or 13 or 14

\#16 dorsalgia.mp.

\#17 exp Back Pain/

\#18 backache.mp.

\#19 ((lumb* adj pain) or (back adj pain)).mp.

\#20 coccyx.mp.

\#21 coccydynia.mp.

\#22 sciatica.mp.

\#23 exp sciatic neuropathy/

\#24 spondylosis.mp.

\#25 lumbago.mp.

\#26 back disorder*.mp

\#27 16 or 17 or 18 or 19 or 20 or 21 or 22 or 23 or 24 or 25 or 26

\#28 4 and 15 and 27

\#29 limit 28 to humans

2930 results 
PsycINFO search strategy on 25 February 2021

ID Search Statement

\#1 control:.tw.

\#2 random:.tw.

\#3 exp treatment/

\#4 1 or 2 or 3

\#5 back pain/

\#6 lumbar spinal cord/

\#7 (low adj back adj pain).mp.

\#8 (back adj pain).mp.

\#9 spinal column/

\#10 (lumbar adj2 vertebra*).mp.

\#11 coccyx.mp.

\#12 sciatica.mp.

\#13 lumbago.mp.

\#14 dorsalgia.mp.

\#15 back disorder*.mp.

\#16 "back (anatomy)"/

\#17 ((disc or disk) adj degenerat*).mp.

\#18 ((disc or disk) adj herniat*).mp.

\#19 ((disc or disk) adj prolapse $\left.{ }^{*}\right)$.mp.

\#20 (failed adj back).mp.

\#21 5 or 6 or 7 or 8 or 9 or 10 or 11 or 12 or 13 or 14 or 15 or 16 or 17 or 18 or 19 or 20

\#22 exp Exercise/ or exercise.mp.

\#23 rehabil ${ }^{*}$.mp.

\#24 endurance.mp.

\#25 training.mp

\$26 trunk stabil*.mp.

\#27 core stabil ${ }^{*}$.mp.

\#28 multifidus.mp.

\#29 transverse abdominis.mp.

\#30 longissimus.mp.

\#31 obliques.mp.

\#32 22 or 23 or 24 or 25 or 26 or 27 or 28 or 29 or 30 or 31

\#33 4 and 21 and 32

\#34 limit 33 to human

1046 results

EMBASE Search Strategy on 25 February 2021

ID Search Statement

\#1 random:.tw.

\#2 placebo:.mp.

\#3 double-blind:.tw.

\#4 1 or 2 or 3

\#5 dorsalgia.mp.

\#6 back pain.mp.

\#7 exp BACKACHE/

\#8 (lumb* adj pain).mp.

\#9 coccyx.mp.

\#10 coccydynia.mp.

\#11 sciatica.mp.

\#12 exp ISCHIALGIA/

\#13 spondylosis.mp.

\#14 lumbago.mp.

\#15 5 or 6 or 7 or 8 or 9 or 10 or 11 or 12 or 13 or 14

\#16 exercise*.mp.

\#17 rehab*.mp.

\#18 lumbar stabil*.mp.

\#19 trunk stabil*.mp.

\#20 core stabil ${ }^{*}$.mp. 


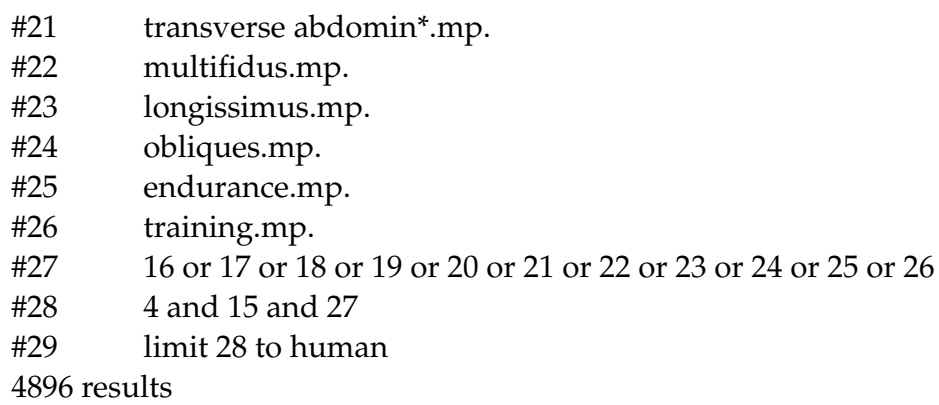

SPORTDiscus search strategy on 25 February 2021

ID Search Statement

\#1 clinical trial

\#2 randomised controlled trial or randomised controlled trial or RCT

\#3 1 or 2

\#4 low back pain or lumbar spine pain or non-specific low back pain or chronic low back pain

\#5 back pain or lumbar pain or spinal pain or backache or lumbago or back injury

\#6 4 or 5

\#7 trunk control or postural control or core stability or trunk stability

\#8 trunk endurance

\#9 trunk exercises

\#10 core stability exercises

\#11 core training

\#12 7 or 8 or 9 or 10 or 11

\#13 3 and 6 and 12

128 results

CINAHL search strategy on 25 February 2021

ID Search Statement

\#1 clinical trial

\#2 randomised controlled trial or randomised controlled trial or RCT

\#3 1 or 2

\#4 low back pain or lumbar spine pain or non-specific low back pain or chronic low back pain

\#5 back pain or lumbar pain or spinal pain or backache or lumbago or back injury

\#6 4 or 5

\#7 trunk control or postural control or core stability or trunk stability

\#8 trunk endurance

\#9 trunk exercises

\#10 core stability exercises

\#11 core training

\#12 7 or 8 or 9 or 10 or 11

\#13 3 and 6 and 12

158 results

\section{Appendix B}


Table A1. Main characteristics of the included randomised controlled trials

\begin{tabular}{|c|c|c|c|c|c|c|}
\hline $\begin{array}{l}\text { First } \\
\text { Author, Year, } \\
\text { Country }\end{array}$ & $\begin{array}{c}\text { Group } \\
\text { Comparison }\end{array}$ & $\begin{array}{c}\text { Participant } \\
\text { Demographics }\end{array}$ & Inclusion Criteria & $\begin{array}{l}\text { Time of } \\
\text { Follow Up }\end{array}$ & Intervention & $\begin{array}{c}\text { Adjusted Treatment Effects } \\
\text { between Group Mean } \\
\text { Change }\end{array}$ \\
\hline $\begin{array}{l}\text { Aasa, 2015, } \\
\text { Sweden }\end{array}$ & $\begin{array}{l}\text { Low-load Motor Control } \\
\text { (LMC) i.e., MC }(\mathrm{n}=35) \\
\text { High Load Lifting (HLL) } \\
\text { i.e., IM }(\mathrm{n}=35)\end{array}$ & $\begin{array}{c}\text { MC: Age } 42 \pm 11 \text { years, } \\
\text { female } \mathrm{n}=19(54 \%) \text {, height } \\
172 \pm 10 \mathrm{~cm} \text {, weight } \\
78 \pm 15 \mathrm{~kg} \\
\text { IM: Age } 42 \pm 10 \text { years, } \\
\text { female } \mathrm{n}=20(57 \%), \text { height } \\
174 \pm 8 \mathrm{~cm} \text {, weight } \\
74 \pm 13 \mathrm{~kg}\end{array}$ & $\begin{array}{l}65 \text { subjects, male and female, } \\
\text { recruited from clinics. Pain } \\
\text { localised to the area of injury / } \\
\text { dysfunction; have a clear, } \\
\text { proportionate mechanical } \\
\text { nature to aggravating and } \\
\text { easing factors; and be } \\
\text { intermittent with } \\
\text { movement/mechanical } \\
\text { provocation }\end{array}$ & 8 weeks & $\begin{array}{l}\text { LMC: Individual and home } \\
\text { practice, progression to } \\
\text { functional movement while } \\
\text { maintaining pain free spine } \\
\text { position. } \\
\text { HLL: group training of } \\
\text { deadlift exercise, progressive } \\
\text { overload. } \\
\text { Both group: } 2 \text { months } \\
\text { intervention, Week } 1-4 \text { have } \\
\text { twice/week, week } 5-8 \text { have } \\
\text { once/week training }\end{array}$ & $\begin{array}{r}\text { NPRS Pre: } 43 \pm 24(22,60) \text {, } \\
\text { change: MC: }-18.5 \pm 26.7 \text {, IM: } \\
-19.0 \pm 25.5 \\
\text { Sorensen test, Pre: MC: } 75 \text { (63, } \\
\text { 87), IM: 87(72, 102); Post: MC: } \\
\text { 87 (74, 99), IM: } 101 \text { (83, 119) } \\
\text { RMDQ: Pre: IM: } 7.2 \pm 4.3, \text { MC: } \\
\text { 7.1 } 1 \text { 3.9; Post: IM: } 3.8 \pm 4.0, \\
\text { MC: } 3.6 \pm 4.2\end{array}$ \\
\hline $\begin{array}{l}\text { Akhtar, 2017, } \\
\text { Pakistan }\end{array}$ & $\begin{array}{c}\text { Core Stabilisation } \\
\text { Exercise i.e., MC }(\mathrm{n}=60) \\
\text { Routine Physiotherapy } \\
\text { i.e., IT }(\mathrm{n}=60)\end{array}$ & $\begin{array}{c}\text { Mixed gender with } \\
\text { unknown ratio } \\
\text { MC: Age } 46.4 \pm 7.4 \text { years, } \\
\text { height } 162 \pm 8 \mathrm{~cm} \text {, weight } \\
64 \pm 10 \mathrm{~kg}, \text { BMI } 24.2 \pm 2.4 \\
\text { IM: Age } 45.5 \pm 6.6 \text { years, } \\
\text { height } 160 \pm 8 \mathrm{~cm} \text {, weight } \\
63.7 \pm 9 \mathrm{~kg}, \text { BMI } 24.8 \pm 3\end{array}$ & $\begin{array}{c}\text { Mechanical CLBP, } 20-60 \text { years, } \\
\text { M \& F, no major spine } \\
\text { pathology, surgery, TB, no } \\
\text { physio intervention within } \\
6 \text { month }\end{array}$ & 6 weeks & $\begin{array}{l}\text { Ultrasound and TENS, } \\
\text { twice/week home training, } \\
\text { once per week physio training } \\
\text { MC: motor control and } \\
\text { dynamic surface and } \\
\text { functional training } \\
\text { Isotonic plus trunk and low } \\
\text { limb stretching }\end{array}$ & $\begin{array}{c}\text { MC: } 7 \text { discontinued. Isotonic: } \\
5 \text { discontinued } \\
\text { NPRS: MC: pre } 5.77 \pm 1.08, \\
\text { post: } 2.69 \pm 0.93 \text {. Isotonic: pre: } \\
5.40 \pm 1.24, \text { post: } 3.69 \pm 0.79\end{array}$ \\
\hline $\begin{array}{l}\text { Areeudomwong, } \\
\text { 2016, Thailand }\end{array}$ & $\begin{array}{c}\mathrm{IM}(\mathrm{n}=21) \\
\text { Control }(\mathrm{n}=21)\end{array}$ & $\begin{array}{c}\text { IM: Age } 35.4 \pm 10.3 \text { years, } \\
\text { female } \mathrm{n}=15(71.4 \%) \text {, height } \\
162.5 \pm 10.5 \mathrm{~cm} \text {, weight } \\
55.6 \pm 7.3 \mathrm{~kg} \\
\text { Con: Age } 36.2 \pm 9.9 \text { years, } \\
\text { female } \mathrm{n}=16(76.2 \%) \text {, height } \\
163.7 \pm 9.4 \mathrm{~cm}, \text { weight } \\
55.8 \pm 8.5 \mathrm{~kg}\end{array}$ & $\begin{array}{c}\text { Male or female }(\mathrm{n}=42) \text {, CLBP, } \\
18-50 \text { years old, at least } 2 \text { on } \\
\text { NRS } \\
\text { Not pregnant, no previous } \\
\text { history of spinal surgery, } \\
\text { neurological deficits, specific } \\
\text { LBP, Cancer, autoimmune } \\
\text { disease }\end{array}$ & 4 weeks & $\begin{array}{c}\text { IM: } 5 \text { times/week, } 3 \text { sets of } \\
15 \text { reps of PNF, } 30 \text { s rest } \\
\text { between set, } 60 \text { s rest between } \\
\text { posture } \\
\text { Control: LBP booklet } \\
\text { including home exercise } \\
\text { prescription and logbook }\end{array}$ & $\begin{array}{c}\text { NPRS: Pre: IM: } 4.08 \pm 1.19, \\
\text { Con: } 4.15 \pm 1.41 ; \text { Post: } \\
1.46 \pm 1.20, \text { Con: } 3.08 \pm 1.50 \\
\text { RMDQ: Pre: IM: } 4.54 \pm 0.78 \\
\text { Con: } 4.85 \pm 1.57 ; \text { Post: IM: } \\
1.69 \pm 0.63, \text { Con: } 3.92 \pm 1.26\end{array}$ \\
\hline
\end{tabular}


Table A1. Cont.

\begin{tabular}{|c|c|c|c|c|c|c|}
\hline $\begin{array}{l}\text { First } \\
\text { Author, Year, } \\
\text { Country }\end{array}$ & $\begin{array}{c}\text { Group } \\
\text { Comparison }\end{array}$ & $\begin{array}{c}\text { Participant } \\
\text { Demographics }\end{array}$ & Inclusion Criteria & $\begin{array}{c}\text { Time of } \\
\text { Follow Up }\end{array}$ & Intervention & $\begin{array}{c}\text { Adjusted Treatment Effects } \\
\text { between Group Mean } \\
\text { Change }\end{array}$ \\
\hline $\begin{array}{l}\text { Areeudomwong, } \\
\text { 2019, Thailand }\end{array}$ & $\begin{array}{l}\text { MC }(n=15) \\
\operatorname{IM}(n=15) \\
\text { IT }(n=15)\end{array}$ & $\begin{array}{c}\text { IM: Age } 25 \pm 8.5 \text { years, } \\
\text { female: } \mathrm{n}=12(80 \%), \mathrm{BMI} \\
22.9 \pm 5 \\
\text { MC: Age } 24.1 \pm 10 \text { years, } \\
\text { female: } \mathrm{n}=11(73 \%), \text { BMI } \\
21.9 \pm 4 \\
\text { IM: Age } 24.4 \pm 10 \text { years, } \\
\text { female: } \mathrm{n}=11(73 \%), \mathrm{BMI} \\
22.6 \pm 4\end{array}$ & $\begin{array}{l}\text { CLBP over } 12 \text { weeks, male or } \\
\text { female, aged 18-50 } \\
\text { No disc herniation, SI joint } \\
\text { dysfunction, neurological } \\
\text { compromise, surgery, } \\
\text { pregnancy, use of other } \\
\text { therapies }\end{array}$ & 4 weeks & $\begin{array}{l}\text { MC: isolated progressing to } \\
\text { functional MC with pressure } \\
\text { and EMG biofeedback } \\
\text { IM: PNF progressing to } \\
\text { combined with upper limb } \\
\text { movement } \\
\text { IT: Ultrasound plus isolated } \\
\text { isotonic training }\end{array}$ & $\begin{array}{l}\text { NPRS: Pre: MC: } 4.13 \pm 0.92, \\
\text { IM: } 4.40 \pm 1.40, \text { IT: } 4.07 \pm 1.28 \text {; } \\
\text { Post: MC: } 1.73 \pm 0.96, \text { IM: } \\
2.07 \pm 0.88, \text { IT: } 3.47 \pm 1.55 \\
\text { RMDQ: Pre: MC: } 4.53 \pm 2.13, \\
\text { IM: } 4.60 \pm 2.17, \text { IT: } 4.47 \pm 2.07 ; \\
\text { Post: MC: } 1.47 \pm 1.60, \text { IM: } \\
\text { 1.93 } \pm 1.79, \text { IT: } 3.93 \pm 2.52\end{array}$ \\
\hline $\begin{array}{c}\text { Bae, 2018, } \\
\text { South Korea }\end{array}$ & $\begin{array}{c}\text { CSE }(n=18) \\
\text { SUE i.e., IT }(n=18)\end{array}$ & $\begin{array}{c}\text { SUE: Age } 32.7 \pm 6.1 \text { years, } \\
\text { male: female }=9: 9, \mathrm{BMI} \\
22.5 \pm 2.3 \\
\text { CSE: Age } 32.4 \pm 10.7 \text { years, } \\
\text { male: female } 11: 7, \text { BMI } \\
\quad 22.8 \pm 2.2\end{array}$ & $\begin{array}{c}\text { CLBP with no leg pain } \\
>3 \text { months, M \& F, NPRS 1-6, } \\
\text { aged 20-60, past low back } \\
\text { pain includes severe pain } \\
\text { severely limiting work and } \\
\text { daily activity for }>2 \text { days, at } \\
\text { least } 2 \times / \text { year } \\
\text { No infection, malignancy, } \\
\text { inflammatory disease, } \\
\text { structural deformity, } \\
\text { neurologic sign, } \\
\text { abdominal/spinal surgery, } \\
\text { pregnancy }\end{array}$ & 4 weeks & $\begin{array}{l}\text { CSE: MC progressing to } \\
\text { functional movement. } \\
\text { IT: machine assisted IT on } \\
\text { trunk flexor } \\
\text { Both: } 30 \text { min session daily } \\
\text { exercise, plus } 3 \text { session/week }\end{array}$ & $\begin{array}{c}\text { NPRS: Pre: IT: } 3.0 \pm 1.3, \mathrm{MC} \\
2.9 \pm 0.8 \text {; Post: IT: } 1.5 \pm 1.3 \\
\text { MC: } 2.1 \pm 0.9 \\
\text { ODI: Pre: IT: } 12.8 \pm 8.2, \mathrm{MC}: \\
14.2 \pm \text { 11.6; Post: IT: } 8.0 \pm 7.7 \\
\text { MC: } 12.0 \pm 10.1 \\
\text { RMDQ: Pre: IT: } 2.4 \pm 1.5, \text { MC: } \\
3.1 \pm 2.9 \text {; Post: IT: } 1.1 \pm 1.5 \\
\text { MC: } 2.4 \pm 2.6\end{array}$ \\
\hline $\begin{array}{l}\text { Barradas, 2015, } \\
\text { Brazil }\end{array}$ & $\begin{array}{l}\mathrm{MC}(\mathrm{n}=5) \\
\operatorname{Con}(\mathrm{n}=5)\end{array}$ & $\begin{array}{c}\text { MC: Age } 28.2 \pm 3.9 \text { years, } \\
\text { BMI } 20.8 \\
\text { Con: Age } 27.5 \pm 3.3 \text { years, } \\
\text { BMI } 21.1\end{array}$ & $\begin{array}{l}\text { Female, } 20-50 \text { years } \\
\text { Exclusion: radiating to lower } \\
\text { limbs, acute low back pain, } \\
\text { cognitive problem, previous } \\
\text { lumbar surgery, cancer, other } \\
\text { low back pain intervention }\end{array}$ & 6 weeks & $\begin{array}{l}\text { Intervention: Progressive MC } \\
\text { training twice/week } \\
\text { Con: No detail }\end{array}$ & $\begin{array}{l}\text { NPRS: Pre: MC: } 7.2 \pm 0.58 \\
\text { Con: } 7.80 \pm 0.49 \text {; Post: MC: } \\
2.60 \pm 1.08, \text { Con: } 7.30 \pm 0.86\end{array}$ \\
\hline
\end{tabular}


Table A1. Cont.

\begin{tabular}{|c|c|c|c|c|c|c|}
\hline $\begin{array}{l}\text { First } \\
\text { Author, Year, } \\
\text { Country }\end{array}$ & $\begin{array}{c}\text { Group } \\
\text { Comparison }\end{array}$ & $\begin{array}{c}\text { Participant } \\
\text { Demographics }\end{array}$ & Inclusion Criteria & $\begin{array}{c}\text { Time of } \\
\text { Follow Up }\end{array}$ & Intervention & $\begin{array}{c}\text { Adjusted Treatment Effects } \\
\text { between Group Mean } \\
\text { Change }\end{array}$ \\
\hline $\begin{array}{l}\text { Bhadauria, } \\
\text { 2017, India }\end{array}$ & $\begin{array}{l}\text { Lumbar stabilisation i.e. } \\
\text { MC }(\mathrm{n}=12 / 15) \\
\text { Dynamic strengthening } \\
\text { i.e. IT }(\mathrm{n}=12 / 14) \\
\text { Pilates i.e. IM } \\
(\mathrm{n}=12 / 15)\end{array}$ & $\begin{array}{c}\text { MC: Age } 32.8 \pm 11 \text { years, } \\
\text { male: female }=50: 50, \mathrm{BMI} \\
21.8 \pm 2.9 \\
\text { IT: Age } 36.7 \pm 11 \text { years, } \\
\text { male: female }=42: 58, \mathrm{BMI} \\
24.7 \pm 4.6 \\
\text { IM: Age } 24.4 \pm 11 \text { years, } \\
\text { male: female }=42: 58, \mathrm{BMI} \\
26 \pm 6.2\end{array}$ & $\begin{array}{c}\text { CLBP > } 3 \text { months, male and } \\
\text { female 20-60 years, } \\
\text { Exclusions: fracture, } \\
\text { osteoporosis, degenerative } \\
\text { change, spine disc prolapse, } \\
\text { bone disorder, arthritis, } \\
\text { tumour, radiculopathy, } \\
\text { myelopathy, past spine } \\
\text { surgery, spine infection, severe } \\
\text { psychiatric disorder }\end{array}$ & 6 weeks & $\begin{array}{l}\text { All: hot packs, TENS, warm } \\
\text { up stretching and cool down } \\
\text { totalling } 60 \text { min. } \\
\text { Lumbar stabilisation: ADIM } \\
\text { focused MC training, also } \\
\text { have bird dog } \\
\text { Dynamic strengthening: IT } \\
\text { focused trunk training, has } \\
\text { bird dog and hip bridge } \\
\text { Pilates: IM trunk muscle } \\
\text { contraction in different } \\
\text { positions, has hip bridge }\end{array}$ & $\begin{array}{c}\text { NPRS: Pre: MC: } 7.17 \pm 1.27, \\
\text { IT: } 6.67 \pm 1.56, \text { IM: } 6.42 \pm 1.00 \text {; } \\
\text { Post: MC: } 1.17 \pm 0.72, \text { IT: } \\
2.00 \pm 1.35, \text { IM: } 1.33 \pm 0.98 \\
\text { ODI: Pre: MC: } 39.75 \pm 10.11 \\
\text { IT: } 37.75 \pm 9.27, \text { IM: } \\
28.17 \pm 13.55 ; \text { Post: MC: } \\
6.92 \pm 2.47, \text { IT: } 23.42 \pm 11.01 \\
\text { IM: } 8.42 \pm 5.14\end{array}$ \\
\hline $\begin{array}{l}\text { Cai, 2017, } \\
\text { Singapore }\end{array}$ & $\begin{array}{l}\operatorname{LL}(\mathrm{n}=28) \\
\mathrm{LE}(\mathrm{n}=28) \\
\mathrm{LS}(\mathrm{n}=28)\end{array}$ & $\begin{array}{c}\text { LL: Age } 28.9 \pm 5.3 \text { years, } \\
\text { BMI } 21.7 \pm 2.4 \\
\text { LE: Age } 26.9 \pm 6.4 \text { years, } \\
\text { BMI } 21.8 \pm 2.4 \\
\text { LS: Age } 26.9 \pm 6.4 \text { years, } \\
\text { BMI } 21.9 \pm 2.4\end{array}$ & $\begin{array}{l}\text { 21-45 year old, M \& F, } \\
\text { 18-25 BMI, CLBP between } \\
3-36 \text { months, running } \\
2-5 \times / \text { week, } 2 \mathrm{~km} \\
\text { min/session, min } 6 \text { month } \\
\text { running history, pain intensity } \\
\text { between } 2-4 \text {, no specific spinal } \\
\text { pathology, no spine surgery }\end{array}$ & 8 weeks & $\begin{array}{c}\text { Both interventions: } \\
\text { twice/week } \\
\text { LE: progressive isometric } \\
\text { extensor training } \\
\text { LS: progressive functional MC }\end{array}$ & $\begin{array}{l}\text { NPRS: LE: Pre: } 3.44 \pm 0.87 \\
\text { Post: } 0.76 \pm 0.78 . \text { LS: Pre: } \\
3.62 \pm 1.13 \text {, Post: } 0.65 \pm 0.56\end{array}$ \\
\hline $\begin{array}{l}\text { Calatayud, } \\
\text { 2020, Spain }\end{array}$ & $\begin{array}{c}\mathrm{IM}(\mathrm{n}=42) \\
\mathrm{MC}(\mathrm{n}=43)\end{array}$ & $\begin{array}{c}\text { IM: Age } 52 \pm 11 \text { years, } \\
\text { height } 164 \pm 10 \mathrm{~cm} \text {, weight } \\
76 \pm 19 \mathrm{~kg} \\
\text { MC: Age } 50 \pm 12 \text { years, } \\
\text { height } 165 \pm 7 \mathrm{~cm} \text {, weight } \\
72 \pm 14 \mathrm{~kg}\end{array}$ & $\begin{array}{l}\text { NSLBP, aged 18-75, M \& F } \\
\text { Exclusion: severe somatic } \\
\text { condition, psychiatric } \\
\text { alteration, neurological } \\
\text { disease, spine surgery, } \\
\text { participation in other } \\
\text { intervention program over } \\
\text { past } 6 \text { months, exercise } \\
\text { contra-indication }\end{array}$ & 8 weeks & $\begin{array}{c}\text { IM: Progressive Strength: } \\
\text { group based, isolated and } \\
\text { functional IM trunk training, } \\
3 \text { times/week } \\
\text { MC: } 2 \times / \text { week group training } \\
\text { for } 3 \text { weeks, followed by home } \\
\text { exercise for } 5 \text { weeks, ADIM in } \\
\text { different position, lumbar and } \\
\text { lower limb stretching }\end{array}$ & $\begin{array}{c}\text { NPRS: Pre: MC: } 6.3 \pm 2, \mathrm{IM}: \\
6.2 \pm \text { 2; Post: MC: } 5.1 \pm 3, \mathrm{IM}: \\
4.3 \pm 2 \\
\text { RMDQ: Pre: MC: } 10.2 \pm 5.52 \\
\text { IM: } 7.75 \pm 5.08 ; \text { Post: } \\
\text { MC:7.9 } \pm 5.35, \text { IM: } 4.97 \pm 4.2 \\
\text { Sorensen Test: Pre: MC: } \\
25.97 \pm 29.93, \text { IM: } 34.61 \pm 28.6 \text {; } \\
\text { Post: } 29.67 \pm 28.06, \mathrm{IM}: \\
79 \pm 58.19\end{array}$ \\
\hline
\end{tabular}


Table A1. Cont.

\begin{tabular}{|c|c|c|c|c|c|c|}
\hline $\begin{array}{c}\text { First } \\
\text { Author, Year, } \\
\text { Country }\end{array}$ & $\begin{array}{c}\text { Group } \\
\text { Comparison }\end{array}$ & $\begin{array}{c}\text { Participant } \\
\text { Demographics }\end{array}$ & Inclusion Criteria & $\begin{array}{c}\text { Time of } \\
\text { Follow Up }\end{array}$ & Intervention & $\begin{array}{c}\text { Adjusted Treatment Effects } \\
\text { between Group Mean } \\
\text { Change }\end{array}$ \\
\hline $\begin{array}{c}\text { Cho, 2015, } \\
\text { South Korea }\end{array}$ & $\begin{array}{l}\text { Lumbar Stabilisation } \\
\text { Exercise i.e., IM } \\
(\mathrm{n}=15) \\
\text { Conservative } \\
\text { treatment }(\mathrm{n}=15)\end{array}$ & $\begin{array}{c}\text { IM: Age } 48.1 \pm 6.9 \text { years, } \\
\text { height } 160.8 \pm 6.3 \mathrm{~cm} \text {, weight } \\
61.9 \pm 9.3 \mathrm{~kg} \\
\text { Con: Age } 44 \pm 6.7 \text { years, } \\
\text { height } 163.6 \pm 8.2 \mathrm{~cm} \text {, weight } \\
60.5 \pm 12.2 \mathrm{~kg}\end{array}$ & Males and females with CLBP & 6 weeks & $\begin{array}{l}\text { IM: Side bridge, dead-bug, } \\
\text { bird-dog, } 3 \text { times/week } \\
\text { Con: hot packs (20 min), TENS } \\
\text { (15 min), Ultrasound ( } 5 \text { min) }\end{array}$ & $\begin{array}{l}\text { ODI: Pre: IM: } 30.1 \pm 12.4 \text {, Con: } \\
\text { 30.4 } \pm 11.7 ; \text { Post: IM: } \\
18.4 \pm 8.3 \text {, Con: } 26.2 \pm 11.9\end{array}$ \\
\hline $\begin{array}{l}\text { Costa 2009, } \\
\text { Australia }\end{array}$ & $\begin{array}{l}\mathrm{MC}(\mathrm{n}=77) \\
\operatorname{Con}(\mathrm{n}=77)\end{array}$ & $\begin{array}{c}\text { MC: Age } 54.6 \pm 13 \text { years, } \\
\text { female } \mathrm{n}=45(58 \%) \text {, height } \\
165 \pm 0.1 \mathrm{~cm} \text {, weight } \\
74.5 \pm 17.5 \mathrm{~kg} \\
\text { Con: Age } 52.8 \pm 12.7 \text { years, } \\
\text { female } \mathrm{n}=48(62 \%) \text {, height } \\
164 \pm 0.1 \mathrm{~cm} \text {, weight } \\
75.9 \pm 15.3 \mathrm{~kg}\end{array}$ & $\begin{array}{l}\text { CLBP at least } 3 \text { months, } \\
\text { 18-80 years, does simple trunk } \\
\text { test, not pregnant, not serious } \\
\text { spine pathology, no nerve root } \\
\text { compromise, no exercise } \\
\text { contraindication, M \& F }\end{array}$ & 6 weeks & $\begin{array}{l}\text { twice/week, } 30 \text { min session } \\
\text { MC: progressive MC training } \\
\text { to functional movement. } \\
\text { Placebo: detuned ultrasound } \\
\text { at same frequency. }\end{array}$ & $\begin{array}{c}\text { NPRS: MC: Pre: } 6.8 \pm 2.1, \\
\text { Post: } 4.6 \pm 2.8 \text {. Placebo: Pre: } \\
\text { 6.6 } \pm \text { 2.0, Post: } 5.6 \pm 2.6 \\
\text { RMDQ: MC: Pre: } 13.3 \pm 5.0, \\
\text { Post: } 9.6 \pm 6.5 . \text { Placebo: Pre: } \\
13.4 \pm 4.9, \text { Post: } 11.9 \pm 5.9\end{array}$ \\
\hline
\end{tabular}


Table A1. Cont.

\begin{tabular}{|c|c|c|c|c|c|c|}
\hline $\begin{array}{l}\text { First } \\
\text { Author, Year, } \\
\text { Country }\end{array}$ & $\begin{array}{c}\text { Group } \\
\text { Comparison }\end{array}$ & $\begin{array}{c}\text { Participant } \\
\text { Demographics }\end{array}$ & Inclusion Criteria & $\begin{array}{c}\text { Time of } \\
\text { Follow Up }\end{array}$ & Intervention & $\begin{array}{c}\text { Adjusted Treatment Effects } \\
\text { between Group Mean } \\
\text { Change }\end{array}$ \\
\hline $\begin{array}{l}\text { Farajzadeh, } \\
\text { 2017, Iran }\end{array}$ & $\begin{array}{c}\mathrm{IM}(\mathrm{n}-15) \\
\mathrm{MC}(\mathrm{n}=15)\end{array}$ & $\begin{array}{c}\text { IM: Age } 23.8 \pm 3.5 \text { years. height } \\
171.8 \pm 8 \mathrm{~cm}, \text { weight } \\
70.5 \pm 10.9 \mathrm{~kg} \\
\text { MC: Age } 20.9 \pm 1.2 \text { years, } \\
\text { height } 171.2 \pm 7 \mathrm{~cm} \text {, weight } \\
69.7 \pm 12.7 \mathrm{~kg}\end{array}$ & $\begin{array}{c}\text { CLBP }>3 \text { months, male and } \\
\text { female } 20-40 \text { years, BMI } 20-25, \\
\text { VAS }<4 . \\
\text { No pain on lower limb, spinal, } \\
\text { abdominal and limb surgery, } \\
\text { postural problem due to } \\
\text { muscular weakness, limb } \\
\text { weakness or pain, neurological } \\
\text { defects, cardiovascular } \\
\text { disease, professional athlete }\end{array}$ & 6 weeks & $\begin{array}{c}\text { IM: } 3 \text { times/week, every other } \\
\text { day, } 30 \text { reps of } 10 \mathrm{~s} \text { of IM trunk } \\
\text { training. } \\
\text { MC: standard MC exercises, } \\
\text { same frequency as IM }\end{array}$ & $\begin{array}{l}\text { NPRS Pre: IM: } 2.953 \pm 0.485 \\
\text { MC: } 2.826 \pm 0.654 ; \text { Post: IM: } \\
2.5 \pm 0.49, \text { MC: } 2.653 \pm 0.787 \\
\text { ODI: Pre: IM: } 25.6 \pm 9.69, \text { MC: } \\
\text { 30.07 } \pm 11.65 ; \text { Post: IM: } \\
22.4 \pm 9.03, \text { MC: } 28 \pm 10.16\end{array}$ \\
\hline $\begin{array}{c}\text { Franca, 2010, } \\
\text { Brazil }\end{array}$ & $\begin{array}{l}\text { SS }(n=15) \\
\text { ST }(n=15)\end{array}$ & $\begin{array}{c}\text { SS: Age } 42.1 \pm 8.2 \text { years, BMI } \\
26.4 \pm 4.5 \\
\text { SS: Age } 41.7 \pm 6.4 \text { years, BMI } \\
26.9 \pm 3.6\end{array}$ & $\begin{array}{l}\text { CLBP more than } 3 \text { months, no } \\
\text { cognitive impairment, no back } \\
\text { surgery, spine infection and } \\
\text { rheumatologic disorder. No } \\
\text { spine exercise within } 3 \text { months }\end{array}$ & 6 weeks & $\begin{array}{l}\text { twice/week, } 30 \text { min sessions. } \\
\text { No other exercise. } \\
\text { SS: MC training } \\
\text { ST: isotonic }\end{array}$ & $\begin{array}{c}\text { NPRS SS: Pre: } 5.94 \pm 1.56, \\
\text { Post: } 0.06 \pm 0.16 . \text { ST: Pre: } \\
\text { 6.49 } \pm 1.48, \text { Post: } 2.89 \pm 1.45 \\
\text { ODI: SS: Pre: } 17.07 \pm 3.99 \\
\text { Post: } 1.80 \pm 1.26 . \text { ST: Pre: } \\
17.27 \pm 3.84, \text { Post: } 8.40 \pm 3.13\end{array}$ \\
\hline
\end{tabular}


Table A1. Cont.

\begin{tabular}{|c|c|c|c|c|c|c|}
\hline $\begin{array}{l}\text { First } \\
\text { Author, Year, } \\
\text { Country }\end{array}$ & $\begin{array}{c}\text { Group } \\
\text { Comparison }\end{array}$ & $\begin{array}{c}\text { Participant } \\
\text { Demographics }\end{array}$ & Inclusion Criteria & $\begin{array}{l}\text { Time of } \\
\text { Follow Up }\end{array}$ & Intervention & $\begin{array}{l}\text { Adjusted Treatment Effects } \\
\text { between Group Mean Change }\end{array}$ \\
\hline $\begin{array}{l}\text { Goldby, 2006, } \\
\text { United } \\
\text { Kingdom }\end{array}$ & $\begin{array}{c}\text { spine stabilisation i.e., } \\
\text { MC }(\mathrm{n}=84) \\
\text { Manual Therapy }(\mathrm{n}=89) \\
\text { Control }(\mathrm{n}=40)\end{array}$ & $\begin{array}{c}\text { MC: Age } 43.4 \pm 10.7 \text { years, } \\
\text { female } n=57(68 \%) \\
\text { Con: Age } 41.5 \pm 13 \text { years, } \\
\text { female } n=27(67.5 \%)\end{array}$ & $\begin{array}{l}\text { Mechanical CLBP lasting at } \\
\text { least } 12 \text { weeks, } 18-65 \text { years } \\
\text { old, not (pregnant, had back } \\
\text { surgery, significant spinal } \\
\text { pathology, exercise } \\
\text { contra-indication) }\end{array}$ & 10 week & $\begin{array}{l}\text { MC: Isolated trunk muscle } \\
\text { focused training, patient } \\
\text { education } \\
\text { Control: patient education }\end{array}$ & $\begin{array}{l}\text { Over } 3 \text { months intervention } \\
6 \text { drop out in MC, } 4 \text { drop out in } \\
\text { MT, } 3 \text { drop out in Control } \\
\text { NPRS SS: Pre: } 4.575 \pm 2.754, \\
\text { Post: } 2.881 \pm 2.814 \text {. Control: Pre: } \\
3.76 \pm 3.643, \text { Post } 3.44 \pm 3.643 \text {. } \\
\text { ODI: SS: Pre: } 40.47 \pm 15.62 \text {, Post: } \\
31.00 \pm 17.07 \text {. Control: Pre: } \\
33.54 \pm 12.21 \text {, Post: } 28.1 \pm 17.34\end{array}$ \\
\hline $\begin{array}{l}\text { Gunay 2014, } \\
\text { Turkey }\end{array}$ & $\begin{array}{l}\text { Classical strength } \\
\text { exercise (CSE) i.e., IT } \\
(\mathrm{n}=32) \\
\text { Muscular endurance } \\
\text { training (MET) i.e., IM } \\
(\mathrm{n}=31)\end{array}$ & $\begin{array}{c}\text { IT: Age } 39.2 \pm 7.4 \text { years, BMI } \\
25.2 \pm 4.5, \text { female } 87.1 \% \\
\text { IM: Age } 40.2 \pm 8.0 \text { years, } \\
\text { BMI } 25.5 \pm 3.9, \text { female } 81.3 \%\end{array}$ & $\begin{array}{l}\text { Age } 20-55 \text { years, male and } \\
\text { female, over } 3 \text { months of } \\
\text { CLBP } \\
\text { Exclude: spine surgery history, } \\
\text { structural deformity, tumour, } \\
\text { exercise contraindication }\end{array}$ & 6 week & $\begin{array}{l}\text { CSE: trunk, shoulder and } \\
\text { hip stretching, dynamic } \\
\text { trunk strength training and } \\
\text { bird-dog } \\
\text { MET: } 5 \text { min walk, stretching, } \\
\text { short duration, and multiple } \\
\text { set and rep isometric hold. } \\
\text { Patient posture education, } \\
3 \text { times/week }\end{array}$ & $\begin{array}{l}\text { ODI: Pre MET } 32.42 \pm 6.49 \text { and } \\
\text { CSE } 33.59 \pm 6.28, \mathrm{p}=0.46 . \\
\text { Post MET } 18.29 \pm 5.21 \text { and CSE } \\
21.09 \pm 5.79, \mathrm{p}=0.04 \\
\text { NPRS: Pre MET } 5.5 \pm 81.36 \text { and } \\
\text { CSE } 5.4 \pm 10.95, \mathrm{p}=0.55 . \text { Post } \\
\text { MET } 2.26 \pm 1.12 \text { and CSE } \\
2.56 \pm 1.01, \mathrm{p}=0.26 \\
\text { Sorensen: pre MET } \\
49.13 \pm 21.92 \text { and CSE } \\
46.6 \pm 23.31, \mathrm{p}=0.66 . \text { Post MET } \\
98.33 \pm 30.11 \text { and CSE } \\
77.03 \pm 29.81, \mathrm{p}=0.01\end{array}$ \\
\hline $\begin{array}{l}\text { Harts, 2008, } \\
\text { Netherlands }\end{array}$ & $\begin{array}{l}\text { High Intensity Training } \\
\text { (HIT) i.e., IT ( } \mathrm{n}=31) \\
\text { Wait List Control (WLC) } \\
\qquad(\mathrm{n}=21)\end{array}$ & $\begin{array}{l}\text { HIT: Age: } 44 \pm 10 \text { years, } \\
\text { Con: Age: } 41 \pm 9 \text { years }\end{array}$ & $\begin{array}{l}\text { 18-54 year old male Dutch } \\
\text { army } \\
\text { over } 12 \text { week LBP } \\
\text { Exclude: spine surgery within } \\
2 \text { years, severe pain while } \\
\text { doing isometric trunk } \\
\text { contraction, and nerve root } \\
\text { symptoms }\end{array}$ & 8 weeks & $\begin{array}{l}\text { HIT: } 2 \text { weeks of twice/week, } \\
\text { then } 6 \text { week of once/week, } \\
\text { high intensity dynamic } \\
\text { trunk training } \\
\text { WLC: wait list }\end{array}$ & $\begin{array}{l}1 \text { missed training from HIT } \\
\text { RMDQ (0-24): Pre: HIT: } \\
6.2 \pm 4.4, \text { HIT-WLC: }-1.4 \\
(-4.0 \text { to } 1.1) \text {, LIT-WLC: } 0.3 \\
(-2.3 \text { to } 2.8 \text { for post-pre } \\
\text { intervention }\end{array}$ \\
\hline
\end{tabular}


Table A1. Cont.

\begin{tabular}{|c|c|c|c|c|c|c|}
\hline $\begin{array}{l}\text { First } \\
\text { Author, Year, } \\
\text { Country }\end{array}$ & $\begin{array}{c}\text { Group } \\
\text { Comparison }\end{array}$ & $\begin{array}{c}\text { Participant } \\
\text { Demographics }\end{array}$ & Inclusion Criteria & $\begin{array}{l}\text { Time of } \\
\text { Follow Up }\end{array}$ & Intervention & $\begin{array}{c}\text { Adjusted Treatment Effects } \\
\text { between Group Mean } \\
\text { Change }\end{array}$ \\
\hline $\begin{array}{l}\text { Kankaanpaa, } \\
\text { 1999, Finland }\end{array}$ & $\begin{array}{c}\text { Active }(\mathrm{n}=30) \\
\text { Passive }(\mathrm{n}=24)\end{array}$ & $\begin{array}{c}\text { IT male age: } 40.7 \pm 8.6 \text { years, } \\
\text { BMI: } 26.3 \pm 2.9 . \text { Female age: } \\
38.9 \pm 8.2 \text { years, BMI: } \\
25.7 \pm 4.3 \\
\text { Con male age: } \\
38.0 \pm 6.9 \text { years, BMI: } \\
24.5 \pm 3.0 . \text { Female age: } \\
40.6 \pm 8.1 \text { years, BMI: } \\
25.7 \pm 3.2\end{array}$ & $\begin{array}{l}\text { NSCLBP with moderate } \\
\text { functional disability more } \\
\text { than } 3 \text { months. No previous } \\
\text { back surgery or serious spinal } \\
\text { pathology. No limb } \\
\text { neurological issue. M \& F }\end{array}$ & 12 weeks & $\begin{array}{l}\text { Active: } 24 \text { session, each } \\
\text { session } 90 \text { min. Group } \\
\text { training } 4-5 \text { per session. IT } \\
\text { trunk training. } \\
\text { Passive: thermal and massage } \\
\text { therapy } 1 \times / \text { week for } \\
1 \text { month. }\end{array}$ & $\begin{array}{c}2 \text { men and } 1 \text { woman drop out } \\
\text { of active, } 2 \text { men and } 5 \text { women } \\
\text { drop out of passive therapy } \\
\text { Men NPRS: } \\
\text { Active: Pre: } 5.41 \pm 1.96 \text {, Post: } \\
3.68 \pm 2.88 \text {. Passive: Pre: } \\
4.28 \pm 2.84 \text {, Post: } 4.49 \pm 2.67 \\
\text { Women NPRS: Active: Pre: } \\
5.61 \pm 2.98 \text {, Post } 2.88 \pm 1.88 \text {. } \\
\text { Passive: Pre: } 5.55 \pm 3.11 \text {, Post } \\
4.18 \pm 2.27\end{array}$ \\
\hline $\begin{array}{l}\text { Kim 2015, } \\
\text { South Korea }\end{array}$ & $\begin{array}{c}\text { CORE }(\mathrm{n}=37) \\
\text { Control }(\mathrm{n}=37)\end{array}$ & $\begin{array}{c}\text { CORE: Age } 29.7 \pm 3.9 \text { years. } \\
\text { height } 161.3 \pm 6.2 \mathrm{~cm}, \\
\text { weight } 56.6 \pm 7.1 \mathrm{~kg} \\
\text { Con: Age } 28.6 \pm 3.2 \text { years, } \\
\text { height } 162.8 .2 \pm 7.8 \mathrm{~cm}, \\
\text { weight } 54.3 \pm 7.6 \mathrm{~kg}\end{array}$ & $\begin{array}{c}\text { Female office workers with } \\
\text { CLBP for over } 3 \text { months, } \\
\text { 20-40 years, Can move w/o } \\
\text { aid } \\
\text { Exclude: history of spine or } \\
\text { lower limb surgery, spine } \\
\text { abnormality, pregnant, no } \\
\text { prior exercise intervention }\end{array}$ & 8 weeks & $\begin{array}{l}\text { Both: TENS } 20 \text { min and hot } \\
\text { packs } 15 \text { min } \\
\text { CORE: } 30 \mathrm{~min}, 5 \times / \text { week, MC } \\
\text { Control: TENS and hot pack }\end{array}$ & $\begin{array}{l}\text { CORE drop out } 10 \text { due to } \\
\text { pharmacotherapy, surgery and } \\
\text { pregnancy. Control drop out } \\
11 \text { due to same reason. } \\
\text { NPRS pre: CORE: } 5.6 \pm 7.9 \\
\text { change at rest CORE: } \\
3.56 \pm 0.59, \text { Control } \\
0.58 \pm 0.52\end{array}$ \\
\hline $\begin{array}{c}\text { Kim, 2020, } \\
\text { South Korea }\end{array}$ & $\begin{array}{l}\text { Stretch }(n=25) \\
\operatorname{IM}(n=25) \\
\text { Con }(n=25)\end{array}$ & $\begin{array}{c}\text { IM: Age } 47.0 \pm 9.5 \text { years, } \\
\text { male: female }=11: 11, \text { BMI } \\
23.7 \pm 1.5 \\
\text { MC: Age } 47.8 \pm 8.5 \text { years, } \\
\text { male: female }=12: 8, \text { BMI } \\
\quad 24.0 \pm 1.1\end{array}$ & $\begin{array}{c}\text { CLBP as diagnosed by } \\
\text { orthopaedist, pain over } \\
3 \text { months. NPRS } \geq 3 \text {. Aged } \\
\text { 30-65. } \\
\text { Exclude: spinal surgery, } \\
\text { ankylosing spondylitis or } \\
\text { rheumatoid arthritis, } \\
\text { spondylolisthesis or } \\
\text { spondylolysis, spine/pelvis } \\
\text { fracture, osteoporosis, } \\
\text { continuous pain medication, } \\
\text { smoking, respiratory/heart } \\
\text { disease }\end{array}$ & 6 weeks & $\begin{array}{l}\text { IM: hip stretch and } \\
\text { strengthening with isometric } \\
\text { trunk contraction for } 30 \text { min. } \\
\text { Con: gentle, sham skin } \\
\text { palpitation }\end{array}$ & $\begin{array}{c}\text { NPRS: Pre: IM: } 6.12 \pm 1.02, \\
\text { Con: } 5.85 \pm 1.16 \text {; Post: IM: } \\
2.37 \pm 0.69, \text { Con: } 2.92 \pm 0.61 \\
\text { ODI: Pre: IM: } 56.91 \pm 6.92 \\
\text { Con: } 58.20 \pm 5.27 ; \text { Post: IM: } \\
30.18 \pm 7.66, \text { Con: } 36.70 \pm 5.12 \\
\text { RMDQ: Pre: IM:11.23 } \pm 2.62 \\
\text { Con: } 11.40 \pm 2.28 ; \text { Post: } \\
3.54 \pm 1.59, \text { Con: } 5.55 \pm 1.82\end{array}$ \\
\hline
\end{tabular}


Table A1. Cont.

\begin{tabular}{|c|c|c|c|c|c|c|}
\hline $\begin{array}{l}\text { First } \\
\text { Author, Year, } \\
\text { Country }\end{array}$ & $\begin{array}{c}\text { Group } \\
\text { Comparison }\end{array}$ & $\begin{array}{c}\text { Participant } \\
\text { Demographics }\end{array}$ & Inclusion Criteria & $\begin{array}{l}\text { Time of } \\
\text { Follow Up }\end{array}$ & Intervention & $\begin{array}{c}\text { Adjusted Treatment Effects } \\
\text { between Group Mean } \\
\text { Change }\end{array}$ \\
\hline $\begin{array}{l}\text { Kofotolis, 2006, } \\
\text { Greece }\end{array}$ & $\begin{array}{c}\operatorname{RST}(\mathrm{n}=28) \\
\operatorname{COI}(\mathrm{n}=28) \\
\text { Control }(\mathrm{n}=30)\end{array}$ & $\begin{array}{c}\text { RST: age } 40.6 \pm 6.4 \text { years, } \\
\text { BMI } 23.7 \pm 1.5 . \\
\text { COI age: } 41.8 \pm 7.7 \text { years, } \\
\text { BMI } 23.7 \pm 1.5 . \\
\text { Con age: } 42.1 \pm 8.4 \text { years, } \\
\text { BMI: } 24.0 \pm 1.1\end{array}$ & $\begin{array}{l}\text { Women. Screening for patients } \\
\text { with known mechanical } \\
\text { nature of LBP } \\
\text { Over } 24 \text { weeks CLBP during } \\
\text { or after activity } \\
\text { No additional physical } \\
\text { therapy during intervention }\end{array}$ & 4 weeks & $\begin{array}{l}\text { Both groups: } 5 \text { times per week, } \\
7-10 \text { min cycling and } \\
\text { stretching warm up. } \\
\text { RST: isometric contraction, } \\
3 \text { sets of } 15 \text { reps } \\
\text { COI: alternating eccentric and } \\
\text { concentric contraction, } 3 \text { sets } \\
\text { of } 15 \text { reps } \\
\text { Con: Active daily living } \\
\text { minus exercise }\end{array}$ & $\begin{array}{c}\text { ODI: Pre: RST: } 34.8 \pm 4.0, \text { COI } \\
36.4 \pm 4.4, \text { con } 34.2 \pm 4.0 \text {. Post: } \\
\text { RST: } 24.6 \pm 5.8, \text { COI } \\
24.8 \pm 6.4, \text { con } 30.8 \pm 7.0 \\
\text { NPRS: Pre: RST: } 2.2 \pm 0.8 \\
\text { COI: } 2.3 \pm 0.5, \text { Con: } 1.9 \pm 0.6 ; \\
\text { Post: RST: } 1.4 \pm 0.4, \text { COI: } \\
1.4 \pm 0.5, \text { Con: } 1.6 \pm 0.4 \\
\text { Sorensen test score only } \\
\text { shown in graphic form }\end{array}$ \\
\hline $\begin{array}{l}\text { Kofotolis, 2008, } \\
\text { Greece }\end{array}$ & $\begin{array}{l}\text { Rhythmic Stabilisation } \\
\text { (RS) }(\mathrm{n}=23) \\
\text { RS + TENS }(\mathrm{n}=23) \\
\text { TENS }(\mathrm{n}=23) \\
\text { Con }(\mathrm{n}=21)\end{array}$ & $\begin{array}{c}\text { RST: age } 41.0 \pm 5.5 \text { years, } \\
\text { BMI } 24.9 \pm 1.2 . \\
\text { Con age: } 42.2 \pm 7.8 \text { years, } \\
\text { BMI } 23.8 \pm 1.7 .\end{array}$ & $\begin{array}{l}92 \text { women, CLBP, pain during } \\
\text { or after activity, sitting or } \\
\text { climbing stairs, No previous } \\
\text { back surgery or serious spinal } \\
\text { pathology. No limb } \\
\text { neurological issue. No past } \\
\text { experience with TENS or RS } \\
\text { therapy }\end{array}$ & 4 weeks & $\begin{array}{l}\text { RS: Isometric trunk training } \\
\text { Placebo: sham TENS }\end{array}$ & $\begin{array}{c}\text { ODI: RS: Pre: } 17.1 \pm 2.5 \text {, Post: } \\
12.6 \pm 3.1 \text {. Placebo: Pre: } \\
15.7 \pm 4.7 \text {, Post: } 16.7 \pm 4.7 \\
\text { NPRS: RS: Pre: } 2.1 \pm 0.8, \text { Post: } \\
\text { 1.6 } \pm 0.4 . \text { Placebo: Pre: } \\
2.1 \pm 0.7, \text { Post: } 2.0 \pm 0.4 \\
\text { Sorensen: RS: Pre: } 80.5 \pm 6.0 \\
\text { post: } 137.0 \pm 6.9 . \text { Placebo: Pre: } \\
79.0 \pm 9.3 \text {, Post: } 79.0 \pm 6.8\end{array}$ \\
\hline $\begin{array}{l}\text { Kofotolis, 2016, } \\
\text { Greece }\end{array}$ & $\begin{array}{c}\text { Pilates }(\mathrm{n}=40) \\
\text { General Strengthening } \\
(\mathrm{GS})(\mathrm{n}=40) \\
\text { Control }(\mathrm{n}=40)\end{array}$ & $\begin{array}{c}\text { Con: age } 42.7 \pm 6.1 \text { years, } \\
\text { BMI } 24.7 \pm 3.8 . \\
\text { Pilates age: } 41.2 \pm 8.5 \text { years, } \\
\text { BMI } 26.6 \pm 3.2 . \\
\text { GS } 39.1 \pm 8.7 \text { years, BMI } \\
23.0 \pm 3.7\end{array}$ & $\begin{array}{c}\text { Female, } 25-65 \text { years old, CLBP } \\
\text { over } 12 \text { weeks, unable to } \\
\text { resume daily activity over past } \\
3 \text { weeks. } \\
\text { Exclusion: acute low back } \\
\text { pain, spinal stenosis or } \\
\text { surgery, inflammation } \\
\text { affecting spine, fracture, } \\
\text { spondylolysis or } \\
\text { spondylolisthesis, genetic } \\
\text { spinal abnormality, pregnancy, } \\
\text { cardiovascular problem, } \\
\text { pelvic girdle pain }\end{array}$ & 8 weeks & $\begin{array}{l}\text { Both therapy: No additional } \\
\text { physiotherapy intervention, } \\
24 \times 1 \text { h session, } 3 \text { times /week, } \\
\text { warm up, stretching } \\
\text { General Strengthening: } \\
\text { Isometric trunk training } \\
\text { Pilates: mixed stretch } \\
\text { isometric and isotonic } \\
\text { Control: No training }\end{array}$ & $\begin{array}{c}37 \text { completed Pilates, } \\
36 \text { completed general } \\
\text { strengthening, } 28 \text { completed } \\
\text { control group. Completed } \\
\text { number used in analysis } \\
\text { RMDQ: GS: Pre: } 12.41 \pm 3.69, \\
\text { Post } 4.88 \pm 1.60 \text {. Control: Pre: } \\
11.28 \pm 5.40 \text {, Post: } 10.09 \pm 4.55\end{array}$ \\
\hline
\end{tabular}


Table A1. Cont.

\begin{tabular}{|c|c|c|c|c|c|c|}
\hline $\begin{array}{l}\text { First } \\
\text { Author, Year, } \\
\text { Country }\end{array}$ & $\begin{array}{c}\text { Group } \\
\text { Comparison }\end{array}$ & $\begin{array}{c}\text { Participant } \\
\text { Demographics }\end{array}$ & Inclusion Criteria & $\begin{array}{c}\text { Time of } \\
\text { Follow Up }\end{array}$ & Intervention & $\begin{array}{c}\text { Adjusted Treatment Effects } \\
\text { between Group Mean } \\
\text { Change }\end{array}$ \\
\hline $\begin{array}{c}\text { Koumantakis, } \\
\text { 2005, UK }\end{array}$ & $\begin{array}{l}\mathrm{MC}(\mathrm{n}=29) \\
\mathrm{IM}(\mathrm{n}=26)\end{array}$ & $\begin{array}{c}\text { MC: } 39.2 \pm 11.4 \text { years, BMI } \\
26.2 \pm 4.2 . \\
\text { IM: } 35.2 \pm 9.7 \text { years, BMI } \\
26.4 \pm 3.2\end{array}$ & $\begin{array}{c}\text { Recurrent CLBP lasting } \\
<6 \text { months within past year, } \\
\text { confirmed with radiograph or } \\
\text { MRI. Onset of current pain } \\
>6 \text { weeks, no description on } \\
\text { subject gender } \\
\text { No past spine surgery, red } \\
\text { flags as defined by Clinical } \\
\text { Standards Advisory Group } \\
\text { (CSAG), spondylolysis, } \\
\text { spondylolisthesis }\end{array}$ & 8 week & $\begin{array}{l}\text { Both: stretching and stationary } \\
\text { bike cycling for 10-15 min. } \\
2 \times / \text { week class, total } \\
45-60 \text { min/session, total } \\
\text { exercise of both group are } \\
\text { equalised, home training } \\
30 \text { min, } 3 \text { times / week, } \\
\text { received patient education } \\
\text { booklet } \\
\text { MC: progressive ADIM } \\
\text { training, } 30-45 \text { min, } \\
\text { individual followed by home } \\
\text { exercise } \\
\text { IM: progressive IM training }\end{array}$ & $\begin{array}{c}\text { NPRS: Pre: MC: } 2.69 \pm 2.06, \\
\text { IM: } 4.02 \pm 2.46 \text {; Post: MC: } \\
1.23 \pm 1.37, \text { IM: } 2.13 \pm 1.73 \\
\text { RMDQ: Pre: MC: } 9.2 \pm 4.6, \text { IM: } \\
11.3 \pm 5.2 \text {; Post: MC: } 5.1 \pm 4.0, \\
\text { IM: } 4.7 \pm 3.5\end{array}$ \\
\hline $\begin{array}{c}\text { Lee, 2016, } \\
\text { South Korea }\end{array}$ & $\begin{array}{l}\text { SEG i.e., IM }(\mathrm{n}=15) \\
\text { CEG i.e., IM + lower } \\
\text { body }(\mathrm{n}=15) \\
\text { Con }(\mathrm{n}=6)\end{array}$ & $\begin{array}{c}\text { SEG: } 42.7 \pm 13.4 \text { years, BMI } \\
24.3 \pm 3.1 . \\
\text { CEG age } 46 \pm 8.1 \text { years, BMI } \\
24.1 \pm 2.6 . \\
\text { Con age } 43.3 \pm 9.9 \text { years, } \\
\text { BMI } 27.9 \pm 4.4\end{array}$ & $\begin{array}{c}\text { BMI }>23, \text { CLBP, no regular } \\
\text { exercise participation in past } \\
6 \text { months. } \\
\text { Gender: Not described } \\
\text { Subject demographics: }\end{array}$ & 12 weeks & $\begin{array}{l}\text { SEG: } 2 \text { times/week, } \\
50 \text { min/session } \\
\text { Intensity 11-16 } \\
\text { Con: No detail }\end{array}$ & $\begin{array}{c}\text { NPRS: Pre: IM: } 3.23 \pm 1.49 \\
\text { Con: } 2.42 \pm 0.92 ; \text { Post: IM: } \\
2.20 \pm 1.13 \text {, Con: } 3.58 \pm 1.72 \\
\text { RMDQ: Pre: IM: } 3.8 \pm 3.7 \\
\text { Con: } 0.8 \pm 1.2 ; \text { Post: IM: } \\
1.1 \pm 0.9, \text { Con: } 1.7 \pm 1.6\end{array}$ \\
\hline $\begin{array}{l}\text { Majiwala, 2017, } \\
\text { India }\end{array}$ & $\begin{array}{l}\text { IM (A) }(n=20) \\
\text { IT (B) }(n=20)\end{array}$ & No detail & $\begin{array}{l}40 \text { subjects, } 20-35 \text { years at } \\
\text { physiotherapy department. } \\
\text { Patients may be screened for } \\
\text { mechanical nature of CLBP, } \\
\text { CLBP more than } 3 \text { months } \\
\text { Exclude: back surgery, injury } \\
\text { and trunk training within last } \\
6 \text { months }\end{array}$ & 4 weeks & $\begin{array}{l}\text { Both groups: TENS and hot } \\
\text { pack } \\
\text { A: isometric flexor, extensor } \\
\text { and lateral training } \\
\text { B: isotonic flexor, extensor and } \\
\text { lateral training }\end{array}$ & $\begin{array}{c}\text { NPRS pre-treatment A } \\
7.05 \pm 1.317, \mathrm{~B} 7.3 \pm 0.979 \\
\mathrm{p}=0.577 . \text { Post treatment A } \\
1.7 \pm 0.657, \mathrm{~B} 1.65 \pm 0.745 \\
\mathrm{p}=0.753 \\
\text { Sorensen test pre-treatment A } \\
2.05 \pm 0.510, \mathrm{~B} 2 \pm 0.726 . \text { Post } \\
\text { treatment A } 4 \pm 0.562, \mathrm{~B} \\
3.55 \pm 0.510, \mathrm{p}=0.036 \\
\text { ODI pre-treatment A } \\
23.25 \pm 2.245, \mathrm{~B} 27.75 \pm 5.884 \\
\text { post-treatment A } 7.5 \pm 1.573 \\
\text { B } 8.8 \pm 3.002, \mathrm{p}=0.159\end{array}$ \\
\hline
\end{tabular}


Table A1. Cont.

\begin{tabular}{|c|c|c|c|c|c|c|}
\hline $\begin{array}{l}\text { First } \\
\text { Author, Year, } \\
\text { Country }\end{array}$ & $\begin{array}{c}\text { Group } \\
\text { Comparison }\end{array}$ & $\begin{array}{c}\text { Participant } \\
\text { Demographics }\end{array}$ & Inclusion Criteria & $\begin{array}{l}\text { Time of } \\
\text { Follow Up }\end{array}$ & Intervention & $\begin{array}{c}\text { Adjusted Treatment Effects } \\
\text { between Group Mean } \\
\text { Change }\end{array}$ \\
\hline $\begin{array}{l}\text { Masharawi } \\
\text { 2013, Israel }\end{array}$ & $\begin{array}{l}\text { Intervention }(n=20) \\
\quad \text { Control }(n=20)\end{array}$ & $\begin{array}{c}\text { MC: } 52.5 \pm 10.6 \text { years, BMI } \\
27.2 \pm 5 . \\
\text { Con: } 53.6 \pm 9.5 \text { years, BMI } \\
26.2 \pm 5.5\end{array}$ & $\begin{array}{c}\text { Female, } 45-65 \text { years, with min } \\
12 \text { week CLBP } \\
\text { Exclude: obvious structural } \\
\text { pathology, overt neurological } \\
\text { signs, or joint inflammatory } \\
\text { disease }\end{array}$ & 4 week & $\begin{array}{c}\text { Intervention: MC (based on } \\
\text { paper in reference), bi-weekly } \\
\text { intervention. } \\
\text { Con: ADL guidance only }\end{array}$ & $\begin{array}{c}\text { NPRS: Pre: MC: } 4.0 \pm 1.43, \\
\text { Con: } 3.91 \pm 1.64 \text {; Post: MC: } \\
\text { 1.68 } \pm \text { 0.82, Con: } 3.88 \pm 1.54 \\
\text { RMDQ: Pre: MC: } 14.21 \pm 5.22, \\
\text { Con: } 14.93 \pm 5.96, \text { Post: MC: } \\
9.31 \pm 5.80, \text { Con: } 14.37 \pm 5.77\end{array}$ \\
\hline $\begin{array}{l}\text { Moreira 2019, } \\
\text { Brazil }\end{array}$ & $\begin{array}{c}\text { IM }(n=42) \\
\text { Con }(n=42)\end{array}$ & $\begin{array}{l}\text { No detail on subject } \\
\text { demographics }\end{array}$ & $\begin{array}{l}\text { No details on the subjects' } \\
\text { inclusion and exclusion } \\
\text { criteria }\end{array}$ & 12 weeks & $\begin{array}{l}\text { IM: Functional training } \\
\text { Con: No Detail }\end{array}$ & $\begin{array}{c}\text { NPRS: Pre: IM: } 6.2 \pm 1.3 \text {, Con: } \\
6.0 \pm 1.7 \text {; Post: IM: } 2.3 \pm 1.9 \\
\text { Con: } 5.5 \pm 1.8 \\
\text { ODI: Pre: IM: } 20.7 \pm 10.8, \text { Con: } \\
25.5 \pm 11.3 \text {; Post: IM: } 9.5 \pm 6.3, \\
\text { Con: } 22.5 \pm 10.5 \\
\text { RMDQ: Pre: IM: } 6.9 \pm 5.0 \\
\text { Con: } 8.4 \pm 5.3 ; \text { Post: IM: } \\
2.8 \pm 2.6, \text { Con: } 7.3 \pm 4.7\end{array}$ \\
\hline
\end{tabular}


Table A1. Cont.

\begin{tabular}{|c|c|c|c|c|c|c|}
\hline $\begin{array}{l}\text { First } \\
\text { Author, Year, } \\
\text { Country }\end{array}$ & $\begin{array}{c}\text { Group } \\
\text { Comparison }\end{array}$ & $\begin{array}{c}\text { Participant } \\
\text { Demographics }\end{array}$ & Inclusion Criteria & $\begin{array}{l}\text { Time of } \\
\text { Follow Up }\end{array}$ & Intervention & $\begin{array}{l}\text { Adjusted Treatment Effects } \\
\text { between Group Mean Change }\end{array}$ \\
\hline $\begin{array}{l}\text { Steele, 2013, } \\
\text { United } \\
\text { Kingdom }\end{array}$ & $\begin{array}{l}\text { FullROM }(\mathrm{n}=12) \\
\text { Control }(\mathrm{n}=9)\end{array}$ & $\begin{array}{c}\text { FullROM age: } \\
46 \pm 12.4 \text { years, BMI: } \\
25.2 \pm 3.15 \\
\text { Con age: } 41.7 \pm 15.1, \mathrm{BMI}: \\
25.94 \pm 4.4\end{array}$ & $\begin{array}{l}\text { Male and female with CLBP } \\
\text { for over } 12 \text { weeks } \\
\text { No contraindication for } \\
\text { exercise, acute LBP, pregnancy, } \\
\text { disc herniation or } \\
\text { neuromuscular complication }\end{array}$ & 12 week & $\begin{array}{l}\text { All group: continued } \\
\text { medication, avoid other } \\
\text { exercise. } \\
\text { FullROM: once/week, } \\
\text { repetitive } 72^{\circ} \text { lumbar } \\
\text { extension with resistance } \\
\text { Control: Do nothing }\end{array}$ & $\begin{array}{c}\text { Discontinued: FullROM: } 1 \text { for } \\
\text { pregnancy, } 1 \text { for poor attendance. } \\
\text { NPRS change: FullROM } \\
-3.03 \pm 2.576, \text { Control } \\
0.671 \pm 1.489, \mathrm{p}<0.05 \\
\text { ODI: FullROM }-18.2 \pm 6.63 \\
\text { LimROM }-12 \pm 5.16, \text { Control } \\
-3 \pm 6.87, \mathrm{p}<0.05\end{array}$ \\
\hline $\begin{array}{l}\text { Shaughnessy, } \\
\text { 2004, Ireland }\end{array}$ & $\begin{array}{c}\mathrm{MC}(\mathrm{n}=23) \\
\text { Control (n=22) }\end{array}$ & $\begin{array}{c}\text { Age: } \mathrm{MC}: 43 \pm 9 \\
\text { Con: } 46 \pm 11 \\
\text { no other demographic } \\
\text { details }\end{array}$ & $\begin{array}{c}\text { Inclusion: male and female } \\
\text { age 20-60 years, CLBP for min } \\
12 \text { weeks. } \\
\text { Exclusion: systemic or } \\
\text { structural pathology, } \\
\text { inflammatory joint disease, } \\
\text { overt neurological signs. }\end{array}$ & 10 weeks & $\begin{array}{l}\text { MC: progressive MC to } \\
\text { adding limb movement, } \\
2 \text { times/week, } \\
\text { Con: Wait list }\end{array}$ & $\begin{array}{l}\text { MC: } 3 \text { drop out, Control } 1 \text { drop } \\
\text { out. } \\
\text { ODI: MC: Pre: } 37 \pm 13 \text {, Post: } \\
26 \pm \text { 14. Control: Pre: } 41 \pm 15 \\
\text { Post: } 44 \pm 16 \\
\text { RMDQ: MC: Pre: } 10.0 \pm 4.1 \text {, Post: } \\
\text { 5.1 } \pm \text { 2.8. Control: Pre: } 10.7 \pm 5.6, \\
\text { Post: } 11.3 \pm 5.6\end{array}$ \\
\hline
\end{tabular}


Table A1. Cont.

\begin{tabular}{|c|c|c|c|c|c|c|}
\hline $\begin{array}{l}\text { First } \\
\text { Author, Year, } \\
\text { Country }\end{array}$ & $\begin{array}{c}\text { Group } \\
\text { Comparison }\end{array}$ & $\begin{array}{c}\text { Participant } \\
\text { Demographics }\end{array}$ & Inclusion Criteria & $\begin{array}{l}\text { Time of } \\
\text { Follow Up }\end{array}$ & Intervention & $\begin{array}{c}\text { Adjusted Treatment Effects } \\
\text { between Group Mean Change }\end{array}$ \\
\hline $\begin{array}{l}\text { You, 2015, } \\
\text { Taiwan }\end{array}$ & $\begin{array}{l}\text { Sling exercise }(\mathrm{n}=9) \\
\quad \text { Control }(\mathrm{n}=7)\end{array}$ & $\begin{array}{c}\text { Sling: age: } 27.6 \pm 6.7 \text { years, } \\
\text { height } 165 \pm 7 \mathrm{~cm} \text {, weight } \\
57.6 \pm 12.2 \mathrm{~kg} \text {. } \\
\text { Con: age: } 27.6 \pm 5.6, \text { height } \\
160 \pm 4 \text {, weight } 57 \pm 9.9 \mathrm{~kg} .\end{array}$ & $\begin{array}{l}\text { CLBP over } 3 \text { months. } \\
\text { Exclude: spondylolisthesis } \\
\text { and disc herniation }\end{array}$ & 6 weeks & $\begin{array}{c}3 \text { times/week, } 10 \text { min warm } \\
\text { up and } 30 \text { min Sling exercise } \\
\text { Con: No exercise }\end{array}$ & $\begin{array}{c}2 \text { drop out in sling and } 2 \text { drop out } \\
\text { in control due to personal reasons } \\
\text { NPRS: Control: Pre } 3.75 \pm 0.87 \\
\text { post } 2.38 \pm 1.03 \text {. Training: Pre } \\
4.29 \pm 1.44, \text { post } 1.33 \pm 1.17 \\
\text { ODI: Control: Pre } \\
19.00 \pm 16.20 \text { post } 18.50 \pm 11.82 \text {. } \\
\text { Training Pre } 14.29 \pm 3.90 \text { post } \\
8.86 \pm 6.41\end{array}$ \\
\hline $\begin{array}{c}\text { Sousa, } 2009, \\
\text { Brazil }\end{array}$ & $\begin{array}{c}\mathrm{MC}(\mathrm{n}=30) \\
\text { Control }(\mathrm{n}=30)\end{array}$ & $\begin{array}{c}\text { MC: age } 45.3 \text { years, male: } \\
\text { female }=10: 20 . \\
\text { Con age } 47.5, \text { male: } \\
\text { female }=7: 23\end{array}$ & $\begin{array}{l}\text { Male and female, mechanical } \\
\text { pain in lumbar spine }>3 \text { month } \\
\text { Exclude: disc herniation, } \\
\text { tumours, infections, } \\
\text { osteoarticular inflammatory } \\
\text { diseases, fibromyalgia } \\
\text { syndrome, chronic obstructive } \\
\text { pulmonary disease, or } \\
\text { vertebral fracture }\end{array}$ & 8 weeks & $\begin{array}{c}\text { Twice/week for, MC with } \\
\text { biofeedback } \\
\text { Use of } 500 \mathrm{mg} \text { paracetamol } \\
\text { every } 6 \text { hrs as necessary on } \\
\text { both group } \\
\text { Control: No treatment }\end{array}$ & $\begin{array}{c}\text { NPRS: Pre: MC: } 4.79 \pm 2.73, \\
\text { Control: } 5.88 \pm 2.99 \text {; Post: } \\
\text { MC:3.35 } \pm 2.48, \text { Control: } \\
4.76 \pm 2.80 \\
\text { RMDQ: Pre: MC: } 9.97 \pm 6.00 \\
\text { Control: } 12.57 \pm 7.30 ; \text { Post: MC: } \\
5.31 \pm 4.79, \text { Control: } 8.16 \pm 6.20\end{array}$ \\
\hline $\begin{array}{l}\text { Oh, 2015, South } \\
\text { Korea }\end{array}$ & $\begin{array}{l}\text { Sling SEG }(n=10) \\
\text { Swiss Ball SBEG } \\
\quad(n=10) \\
\text { Control }(n=10)\end{array}$ & $\begin{array}{c}\text { SEG age } 46.2 \pm 3.22 \text { years, } \\
\text { height } 170.1 \pm 4.5 \mathrm{~cm}, \\
\text { weight } 71.0 \pm 10.5 \mathrm{~kg} . \\
\text { SBEG age: } 46.0 \pm 3.4 \text { years, } \\
\text { height } 172.0 \pm 3.2 \mathrm{~cm}, \\
\text { weight } 69.3 \pm 9.2 \mathrm{~kg} \\
\text { Con age: } 44.7 \pm 2.7 \text { years, } \\
\text { height } 169.9 \pm 4.9 \mathrm{~cm}, \\
\text { weight } 69.2 \pm 7.8 \mathrm{~kg}\end{array}$ & $\begin{array}{l}\text { Working aged men with } \\
\text { normal BMI with CLBP }\end{array}$ & 12 week & $\begin{array}{c}\text { SBEG and SEG: isometric } \\
\text { trunk holding with limb in } \\
\text { various poses. } 30 \mathrm{~min} / \mathrm{session}, \\
5 \text { times/week. } \\
\text { Con: active daily living }\end{array}$ & $\begin{array}{l}\text { NPRS: Pre: SBEG: } 7.0 \pm 0.9, \text { SEG: } \\
7.1 \pm 1.6, \text { Con: } 6.0 \pm 0.9 \text {; Post: } \\
\text { SBEG: } 5.2 \pm 1.0, \text { SEG: } 4.5 \pm 1.3, \\
\text { Con: } 5.4 \pm 0.9 \\
\text { ODI (out of } 100 \text { ): Pre: SBEG: } \\
28.0 \pm 6.3 \text {, SEG: } 32.4 \pm 6.7 \text {, Con: } \\
18.9 \pm 7.0 . \text { Post: } 13.8 \pm 6.3, \text { SEG: } \\
10.4 \pm 4.2, \text { Con: } 14.5 \pm 4.7\end{array}$ \\
\hline $\begin{array}{l}\text { Yi, 2008, South } \\
\text { Korea }\end{array}$ & $\begin{array}{c}\operatorname{IM}(\mathrm{n}=20) \\
\operatorname{IT}(\mathrm{n}=20) \\
\mathrm{IM}+\mathrm{IT}(\mathrm{n}=20)\end{array}$ & $\begin{array}{l}\text { IM age } 51.6 \pm 9.4 \text { years, } \\
\text { male: female }=2: 12 \\
\text { IT age } 50.2 \pm 10.5 \text { years, } \\
\text { male: female }=2: 15\end{array}$ & $\begin{array}{c}\text { CLBP }>6 \text { months, } 20-60 \text { years } \\
\text { old } \\
\text { Exclude: lower limb radiating } \\
\text { pain, pregnant, artificial joint }\end{array}$ & 8 weeks & No details & $\begin{array}{c}\text { ODI: Pre: IM: } 27.9 \pm 15.5, \text { IT: } \\
29.5 \pm \text { 12.6; Post: IM: } 14.1 \pm 8.9 \\
\text { IT: } 16.6 \pm 11.7 \\
\text { NPRS: Pre: IM: } 5.01 \pm 2.92, \text { IT: } \\
5.39 \pm 2.90 \text {; Post: IM: } 1.46 \pm 1.45 \\
\text { IT: } 1.75 \pm 1.63\end{array}$ \\
\hline
\end{tabular}


Table A1. Cont.

\begin{tabular}{|c|c|c|c|c|c|c|}
\hline $\begin{array}{l}\text { First } \\
\text { Author, Year, } \\
\text { Country }\end{array}$ & $\begin{array}{c}\text { Group } \\
\text { Comparison }\end{array}$ & $\begin{array}{c}\text { Participant } \\
\text { Demographics }\end{array}$ & Inclusion Criteria & $\begin{array}{c}\text { Time of } \\
\text { Follow Up }\end{array}$ & Intervention & $\begin{array}{c}\text { Adjusted Treatment Effects } \\
\text { between Group Mean } \\
\text { Change }\end{array}$ \\
\hline $\begin{array}{l}\text { Shamsi 2015, } \\
\text { Iran }\end{array}$ & $\begin{array}{l}\mathrm{MC}(\mathrm{n}=19) \\
\mathrm{IM}(\mathrm{n}=20)\end{array}$ & $\begin{array}{c}\text { MC: age } 38.5 \pm 11.9 \text { years, } \\
\text { male: female }=6: 13, \text { height } \\
166.7 \pm 8.6 \mathrm{~cm} \text {, weight } \\
\quad 68.9 \pm 15.7 \mathrm{~kg} \\
\text { IM: age } 47.7 \pm 10.4 \text { years, } \\
\text { male: female }=6: 14, \text { height } \\
163.7 \pm 8.3 \mathrm{~cm}, \text { weight } \\
73.1 \pm 8.9 \mathrm{~kg}\end{array}$ & $\begin{array}{c}\text { Male and female with CLBP } \\
>3 \text { months, aged 18-60 years, } \\
\text { NPRS 3-6 } \\
\text { Exclude: lower limb pathology or } \\
\text { anomaly, inflammatory diseases, } \\
\text { osteoporosis, arthritis, bone } \\
\text { disease }\end{array}$ & 6 weeks & $\begin{array}{c}3 \text { times/week } \\
\text { intensity equalised }\end{array}$ & $\begin{array}{c}\text { NPRS: Pre: MC: } 5.24 \pm 0.92, \\
\text { IM: } 5.30 \pm \text { 0.92; Post: MC: } \\
\text { 1.59 } \pm 1.24, \text { IM: } 1.49 \pm 1.41 \\
\text { ODI: Pre: MC: } 51.1 \pm 12.7, \text { IM: } \\
49.8 \pm 10.8 ; \text { Post: MC: } \\
33.3 \pm 11.0, \text { IM: } 37.4 \pm 11.1\end{array}$ \\
\hline $\begin{array}{l}\text { Segal-Snir 2016, } \\
\text { Israel }\end{array}$ & $\begin{array}{l}\text { Intervention }(\mathrm{n}=25) \\
\quad \text { Control }(\mathrm{n}=20)\end{array}$ & $\begin{array}{c}\text { IT: age } 57.2 \pm 8.4 \text { years, BMI } \\
27.6 \pm 4.5 \\
\text { Con: age } 54.7 \pm 6.5 \text { years, } \\
\text { BMI } 28.5 \pm 5.5\end{array}$ & $\begin{array}{l}45 \text { female aged } 40-70 \text { years, CLBP } \\
\quad>12 \text { weeks. } \\
\text { Exclude: systemic or structural } \\
\text { pathology, inflammatory joint } \\
\text { disease or neurological signs. }\end{array}$ & & $\begin{array}{c}\text { Control: Wait list } \\
\text { Intervention: } 40 \mathrm{~min} / \mathrm{session}, \\
2 \text { times/week, } 4 \text { week } \\
\text { duration, } 10 \text { person/group } \\
\text { max, } 5 \text { position IT exercises. }\end{array}$ & $\begin{array}{r}\text { NPRS: Pre: IT: } 7 \pm 2.3 \text {, Con: } \\
8 \pm 1.4 \text {; Post: IT: } 7 \pm 3 \text {, Con: } \\
7 \pm 1.9 \\
\text { RMDQ: Pre: IT: } 13 \pm 6.2 \text {, Con: } \\
14 \pm 6.3 \text {; Post: IT: } 11 \pm 6.4 \\
\text { Con: } 14 \pm 6.3\end{array}$ \\
\hline $\begin{array}{l}\text { Noormo- } \\
\text { hammadpour } \\
\text { 2018, Iran }\end{array}$ & $\begin{array}{l}\text { Intervention }(\mathrm{n}=18) \\
\quad \text { Control }(\mathrm{n}=18)\end{array}$ & $\begin{array}{c}\text { MC age } 43.3 \pm 7.5 \text { years, } \\
\text { BMI } 24.0 \pm 1.7 \\
\text { Con age } 41.3 \pm 6.4 \text { years, } \\
\text { BMI } 24.3 \pm 2.1\end{array}$ & $\begin{array}{c}\text { Female nurses with CLBP } \\
>3 \text { months in past } 6 \text { months, aged } \\
18-55, \\
\text { Exclude: spine/abdominal } \\
\text { trauma/surgery, systemic disease, } \\
\text { spine deformity, abdominal wall } \\
\text { hernia, participation in core } \\
\text { stability intervention }\end{array}$ & 8 weeks & $\begin{array}{l}\text { Intervention: } 2 \text { floor and } \\
2 \text { swiss ball exercises, ADIM } \\
\text { focused, progressing to } \\
\text { functional movement, home } \\
\text { exercise } 3 \text { times/day for } \\
10 \text { reps } \\
\text { Con: Wait list }\end{array}$ & $\begin{array}{l}\text { NRPS: Pre: MC: } 5.18 \pm 2.41 \\
\text { Con: } 4.42 \pm 2.65 ; \text { Post: MC: } \\
\text { 1.24 } \pm 1.35 \text {, Con: } 3.94 \pm 2.09 \\
\text { RMDQ: Pre: MC: } 7.8 \pm 3.4 \\
\text { Con: } 9.5 \pm 4.9 ; \text { Post: MC: } \\
1.7 \pm 2.4 \text {, Con: } 7.9 \pm 3.3\end{array}$ \\
\hline $\begin{array}{l}\text { Rathod 2015, } \\
\text { Pakistan }\end{array}$ & $\begin{array}{l}\mathrm{MC}(\mathrm{n}=20) \\
\mathrm{IM}(\mathrm{n}=20)\end{array}$ & No demographic data & $\begin{array}{l}\text { Male and female clerks with } \\
\text { CLBP }>3 \text { months, age } 30-45 \text { years } \\
\text { Exclude: lumbar spondylolysis, } \\
\text { spondylolisthesis, acute disc } \\
\text { prolapse, neurological disorders, } \\
\text { other muscoskeletal disorders, } \\
\text { hypertension and ischaemic heart } \\
\text { disease }\end{array}$ & 4 week & $\begin{array}{l}\text { Both: } 20 \text { min ultrasound first } \\
10 \text { days, } \\
\text { MC: Progressive trunk } \\
\text { training with ADIM with help } \\
\text { of biofeedback unit } \\
\text { IM: IM trunk training, 5-10 s } \\
\text { hold, 5-20 reps }\end{array}$ & $\begin{array}{l}\text { NPRS: Pre: MC: } 7.30 \pm 0.97 \\
\text { IM: } 6.80 \pm 1.36 \text {; Post: MC: } \\
0.25 \pm 0.78, \text { IM: } 1.20 \pm 1.10 \\
\text { ODI (0-100): Pre: MC: } \\
\text { 45.76 } \pm \text { 7.81, IM: } 46.67 \pm 6.55 ; \\
\text { Post: MC: } 8.21 \pm 7.94, \text { IM: } \\
\text { 24.86 } \pm 7.20\end{array}$ \\
\hline
\end{tabular}


Table A1. Cont.

\begin{tabular}{|c|c|c|c|c|c|c|}
\hline $\begin{array}{c}\text { First } \\
\text { Author, Year, } \\
\text { Country }\end{array}$ & $\begin{array}{c}\text { Group } \\
\text { Comparison }\end{array}$ & $\begin{array}{c}\text { Participant } \\
\text { Demographics }\end{array}$ & Inclusion Criteria & $\begin{array}{l}\text { Time of } \\
\text { Follow Up }\end{array}$ & Intervention & $\begin{array}{c}\text { Adjusted Treatment Effects } \\
\text { between Group Mean Change }\end{array}$ \\
\hline $\begin{array}{l}\text { Shamsi 2016, } \\
\text { Iran }\end{array}$ & $\begin{array}{l}\mathrm{MC}(\mathrm{n}=24) \\
\mathrm{IM}(\mathrm{n}=24)\end{array}$ & $\begin{array}{c}\text { MC age } 39.2 \pm 11.7 \text { years, } \\
\text { male: female }=7: 15, \text { height } \\
166.4 \pm 9.1 \mathrm{~cm} \text {, weight } \\
70.1 \pm 15.1 \mathrm{~kg} \\
\text { IM age } 47.9 \pm 10.2 \text { years, male: } \\
\text { female }=6: 15, \text { height } \\
163.7 \pm 8.1 \mathrm{~cm}, \text { weight } \\
74.3 \pm 10.5 \mathrm{~kg}\end{array}$ & $\begin{array}{c}\text { CLBP }>3 \text { months, VAS 3-6, } \\
18-60 \text { years. } \\
\text { Exclude: lumbar radicular } \\
\text { pain, lower limb pathology } \\
\text { or anomaly }\end{array}$ & 6 weeks & $\begin{array}{l}\text { Both: stretching } 8 \text { min, stationary } \\
\text { cycling } 5 \text { min, progressive } \\
\text { exercise, group training, } 3 \times \text { per } \\
\text { week } \\
\text { MC: } 20 \text { min trunk training, } \\
\text { focused on ADIM } \\
\text { IM: } 14 \text { min trunk training, focused } \\
\text { on extensor and flexor muscles }\end{array}$ & $\begin{array}{c}\text { NPRS: Pre: MC: } 5.14 \pm 0.98, \text { IM: } \\
\text { 5.07 } \pm \text { 1.13; Post: MC: } 1.51 \pm 1.18 \\
\text { IM: } 1.51 \pm 1.38 \\
\text { ODI (0-100): Pre: MC: } 50.5 \pm 12.1 \text {, } \\
\text { IM: } 50.7 \pm 11.3 \text {; Post: MC: } \\
32.8 \pm 10.5, \text { IM: } 37.6 \pm 10.9 \\
\text { Sorensen: Pre: MC: } 70.6 \pm 57.1 \\
\text { IM: } 80.9 \pm 48.6 \text {; Post: MC: } \\
\text { 117.2 } \pm 60.2, \text { IM: } 113.2 \pm 52.0\end{array}$ \\
\hline $\begin{array}{c}\text { Shamsi, 2020, } \\
\text { Iran }\end{array}$ & $\begin{array}{c}\text { Core Stability i.e., } \\
\text { MC }(\mathrm{n}=28) \\
\text { General Exercise } \\
\text { i.e., IM }(\mathrm{n}=28)\end{array}$ & $\begin{array}{c}\text { MC age } 38.9 \pm 12.2 \text { years, } \\
\text { male: female }=11: 16, \text { height } \\
167.6 \pm 8.8 \mathrm{~cm}, \text { weight } \\
71.9 \pm 14.2 \mathrm{~kg} \\
\text { IM age } 47.0 \pm 9.9 \text { years, male: } \\
\text { female }=7: 17, \text { height } \\
164.0 \pm 9.1 \mathrm{~cm}, \text { weight } \\
74.2 \pm 10.7\end{array}$ & $\begin{array}{l}\text { Male and female, CLBP } \\
\text { based on imaging and pain } \\
\text { provocation, duration } \\
>3 \text { months, NPRS }=3-6, \\
\text { aged } 18-60, \text { M \& F } \\
\text { Exclude: spine and lower } \\
\text { limb pathology or anomaly }\end{array}$ & 6 week & $\begin{array}{l}\text { Both: } 8 \text { min stretching and } 5 \text { min } \\
\text { stationary cycling, } 3 \times / \text { week } \\
\text { Core Stability: MC training, } \\
\text { focused on ADIM and isolated } \\
\text { deep muscle contraction } \\
\text { General Exercise: Isolated floor } \\
\text { IM trunk training based on } \\
\text { description of (Shamsi 2015) }\end{array}$ & $\begin{array}{c}\text { ODI (0-100): Pre: MC: } \\
\text { 50.55 } \pm \text { 12.08, IM: } 50.67 \pm 10.41 \\
\text { Post: MC: } 32.77 \pm 11.0, \text { IM: } \\
\text { 37.62 } \pm 10.87 \\
\text { NPRS: Pre: MC: } 5.136 \pm 0.902, \text { IM: } \\
\text { 5.286 } \pm 0.902 ; \text { Post: MC: } \\
1.509 \pm 1.24, \text { IM: } 1.510 \pm 1.380\end{array}$ \\
\hline $\begin{array}{l}\text { Cruz-Diaz } \\
\text { 2017, Spain }\end{array}$ & $\begin{array}{l}\text { Mat Pilates group } \\
\text { i.e., } \mathrm{MC}(\mathrm{n}=34) \\
\text { Con }(\mathrm{n}=30)\end{array}$ & $\begin{array}{c}\text { MC age } 36.9 \pm 12.5 \text { years, } \\
\text { male: female }=11: 23 \text {, height } \\
167.6 \pm 8.8 \mathrm{~cm} \text {, weight } \\
71.9 \pm 14.2 \mathrm{~kg} \\
\text { Con age } 36.3 \pm 10.7 \text { years, } \\
\text { male: female }=13: 21 \text {, height } \\
164.0 \pm 9.1 \mathrm{~cm} \text {, weight } \\
74.2 \pm 10.7 \mathrm{~kg}\end{array}$ & $\begin{array}{c}\text { CLBP > } 12 \text { weeks, age } 18-50, \\
\text { NPRS } 3-7 \\
\text { Exclude: radiculopathy, } \\
\text { fracture, stenosis, tumour, } \\
\text { Pilates or physical therapy } \\
\text { training over past } 6 \text { months. } \\
\text { Not pregnant. }\end{array}$ & 12 weeks & $\begin{array}{l}\text { Pilates Mat Group: warm up, } \\
\text { training and cool down. Focus on } \\
\text { pelvic tilt and ADIM, joint } \\
\text { mobility drills. Training in group } \\
\text { of } 4 \text {. } \\
\text { Control: Not described }\end{array}$ & $\begin{array}{c}\text { NPRS: Pre: MC: } 4.64 \pm 1.22, \text { Con: } \\
4.84 \pm 1.04 \text {; Post: MC: } 2.1 \pm 1.36 \\
\text { Con: } 4.96 \pm 1.31 \\
\text { RMDQ: Pre: MC: } 11.38 \pm 5.02 \\
\text { Con: } 10.50 \pm 4.89 ; \text { Post: MC: } \\
6.35 \pm 5.3 \text {, Con: } 10.41 \pm 5.6\end{array}$ \\
\hline $\begin{array}{l}\text { Kim 2017, } \\
\text { South Korea }\end{array}$ & $\begin{array}{l}\text { PNF i.e., IM } \\
\quad(n=15) \\
\text { Con }(n=15)\end{array}$ & $\begin{array}{c}\text { IM age } 39.8 \pm 5.5 \text { years, male: } \\
\text { female }=8: 7, \text { height } \\
168.7 \pm 7.3 \mathrm{~cm} \text {, weight } \\
67.6 \pm 9.5 \mathrm{~kg} . \\
\text { Con age } 39.4 \pm 5.7 \text { years, male: } \\
\text { female } 9: 6, \text { height } \\
168.7 \pm 8.0 \mathrm{~cm} \text {, weight } \\
67.1 \pm 9.7 \mathrm{~kg} .\end{array}$ & $\begin{array}{l}\text { CLBP }>12 \text { weeks, age } \\
30-40 \text { years, no } \\
\text { exercise/mental problem, } \\
\text { neurologic sensation, } \\
\text { muscular paralysis. }\end{array}$ & 6 weeks & $\begin{array}{l}\text { PNF: isometric holding in } \\
\text { different position, resisting force } \\
\text { in different direction while } \\
\text { maintaining normal breathing. } \\
\text { Control: no description } \\
5 \text { times / weeks }\end{array}$ & $\begin{array}{l}\text { NPRS: Pre: IM: } 6.6 \pm 1.12, \text { Con: } \\
6.5 \pm 1.11 \text {; Post: IM: } 2.4 \pm 0.54 \\
\text { Con: } 4.6 \pm 0.90 . \\
\text { ODI: Pre: IM: } 33.3 \pm 6.8, \text { Con: } \\
33.5 \pm 4.8 \text {; Post: IM: } 21.3 \pm 3.4 \\
\text { Con: } 28.9 \pm 4.6\end{array}$ \\
\hline
\end{tabular}




\section{Appendix C}

Appendix C.1. Sensitivity Analysis of the Pairwise Meta-Analyses with the Removal of Randomised Controlled Trials with a High Risk of Bias

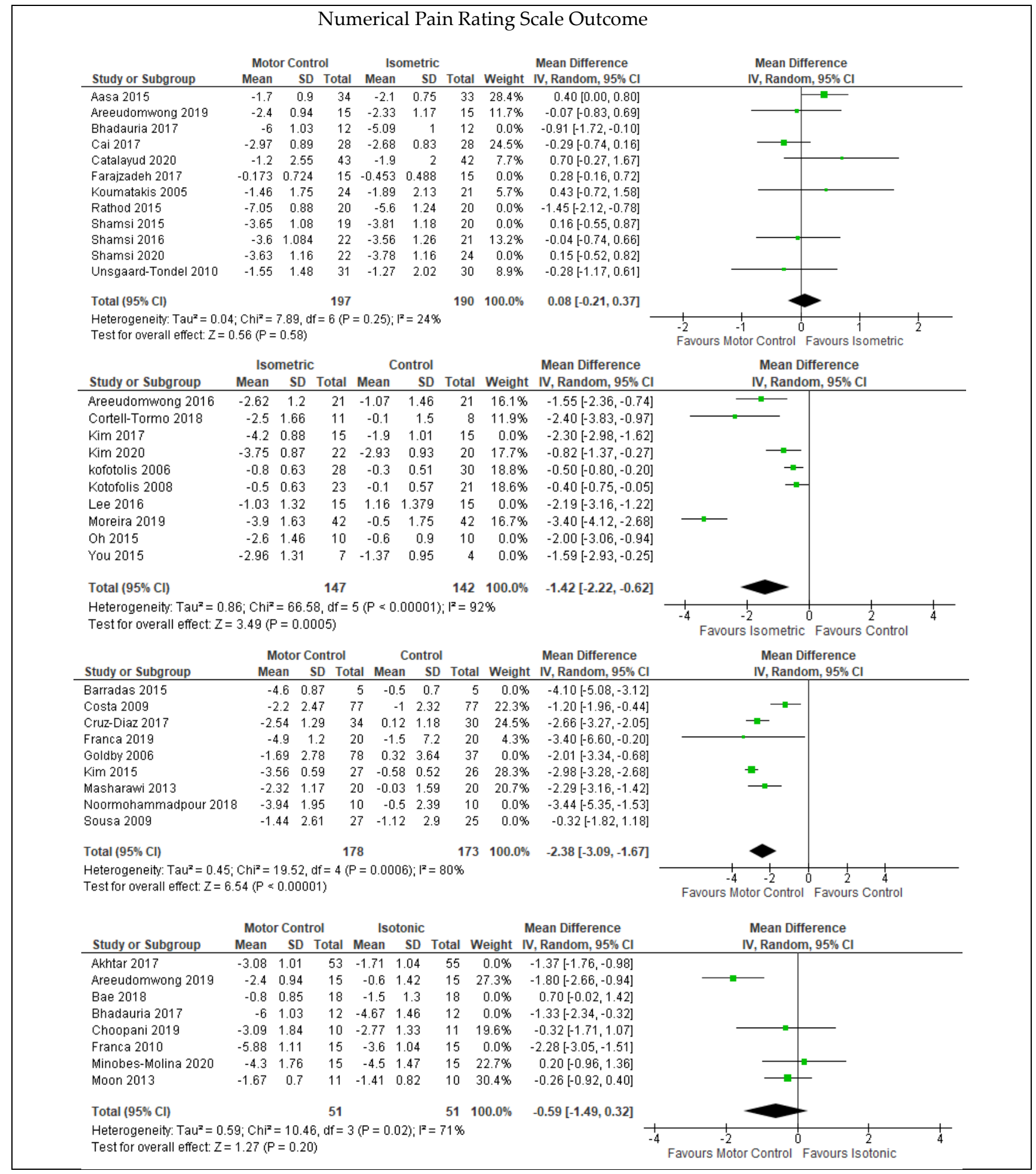




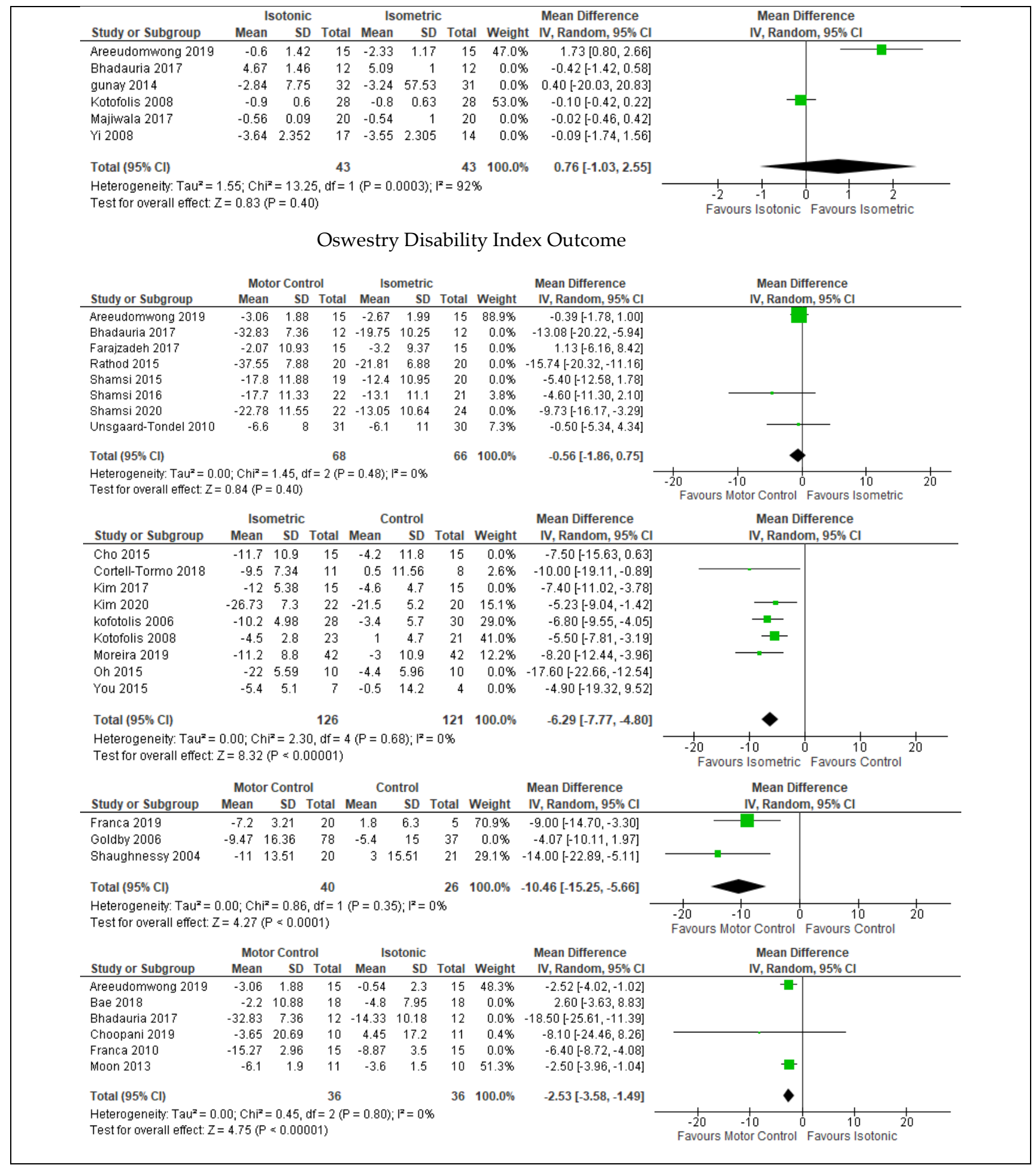




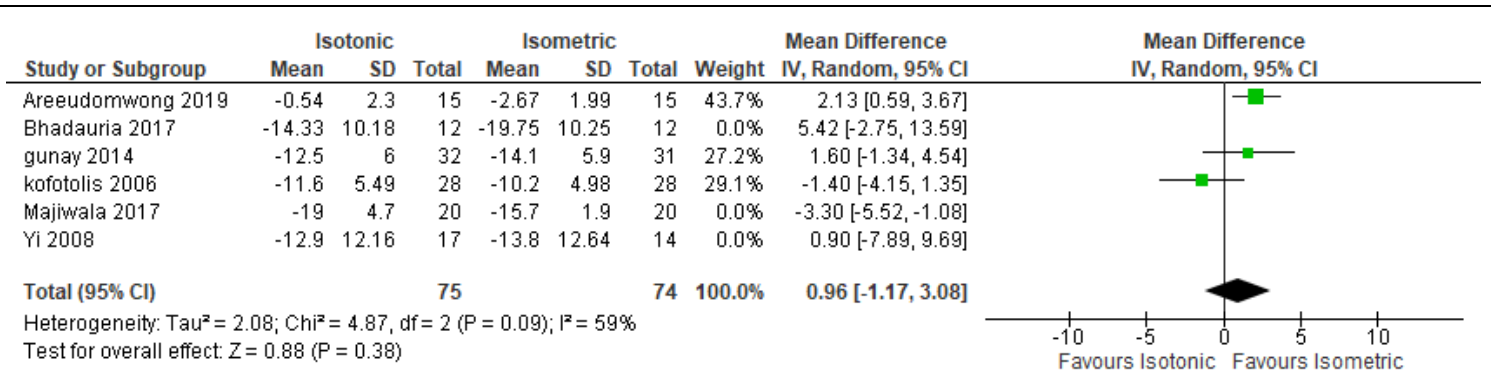

Roland Morris Disability Questionnaire Outcome

\begin{tabular}{|c|c|c|c|c|c|c|c|c|c|c|c|}
\hline \multirow[b]{2}{*}{ Study or Subgroup } & \multicolumn{3}{|c|}{ Motor Control } & \multicolumn{3}{|c|}{ Control } & \multicolumn{2}{|r|}{ Mean Difference } & \multirow{2}{*}{\multicolumn{3}{|c|}{$\begin{array}{l}\text { Mean Difference } \\
\text { IV, Random, } 95 \% \mathrm{Cl}\end{array}$}} \\
\hline & Mean & SD & Total & Mean & SD & Total & Weight & IV, Random, $95 \% \mathrm{Cl}$ & & & \\
\hline Costa 2009 & -3.7 & 5.8 & 77 & -1.5 & 5.4 & 77 & $35.8 \%$ & $-2.20[-3.97,-0.43]$ & $\longrightarrow$ & & \\
\hline Cruz-Diaz 2017 & -5.03 & 5.16 & 34 & -0.09 & 5.26 & 30 & $25.2 \%$ & $-4.94[-7.50,-2.38]$ & & & \\
\hline Masharawi 2013 & -4.9 & 5.5 & 20 & -0.56 & 5.9 & 20 & $16.7 \%$ & $-4.34[-7.88,-0.80]$ & & & \\
\hline Noormohammadpour 2018 & -6.1 & 2.94 & 10 & -1.6 & 4.18 & 10 & & Not estimable & & & \\
\hline Shaughnessy 2004 & -4.9 & 3.5 & 20 & 0.6 & 5.6 & 21 & $22.3 \%$ & $-5.50[-8.34,-2.66]$ & & & \\
\hline Sousa 2009 & -4.66 & 5.42 & 27 & -4.41 & 6.77 & 25 & $0.0 \%$ & $-0.25[-3.60,3.10]$ & & & \\
\hline Total $(95 \% \mathrm{Cl})$ & & & 151 & & & 148 & $100.0 \%$ & $-3.98[-5.70,-2.27]$ & & & \\
\hline $\begin{array}{l}\text { Heterogeneity: } \operatorname{Tau}^{2}=1.32 ; \mathrm{C} \\
\text { Test for overall effect: } Z=4.56\end{array}$ & $\begin{array}{l}F^{2}=5.32 \\
(P<0.0\end{array}$ & $\begin{array}{l}d f=3 \\
0001)\end{array}$ & $3(P=0$ & $15) ; 1^{2}=$ & $=44 \%$ & & & & $\begin{array}{ccc} & 1 \\
-10 & -5 & 0 \\
& \text { Favours Motor Control }\end{array}$ & Favours C & $\begin{array}{l}5 \\
5 \\
\text { Control }\end{array}$ \\
\hline
\end{tabular}

Appendix C.2. Subgroup Analysis of Isometric and Motor Control Interventions Based on Training Duration

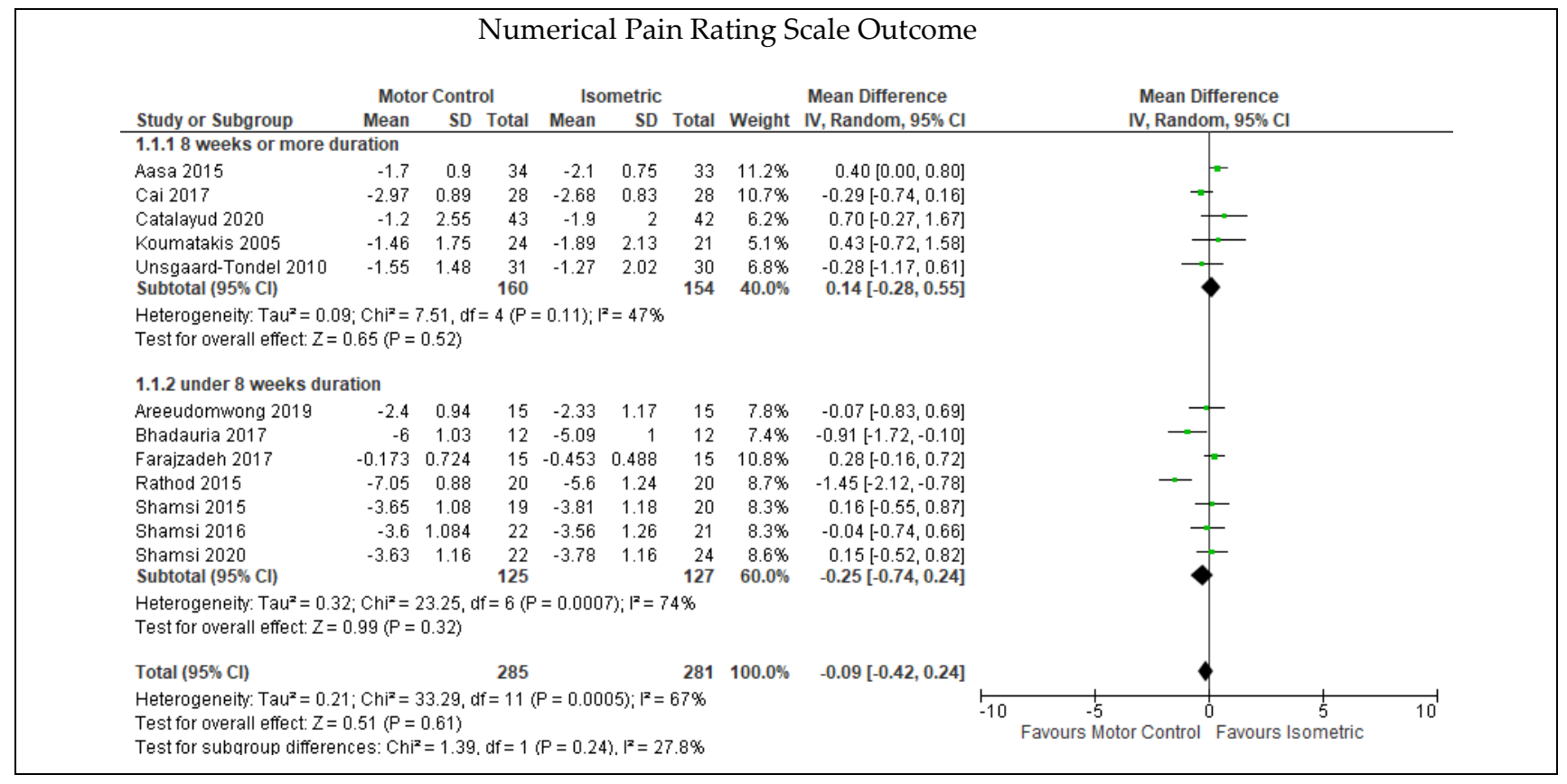




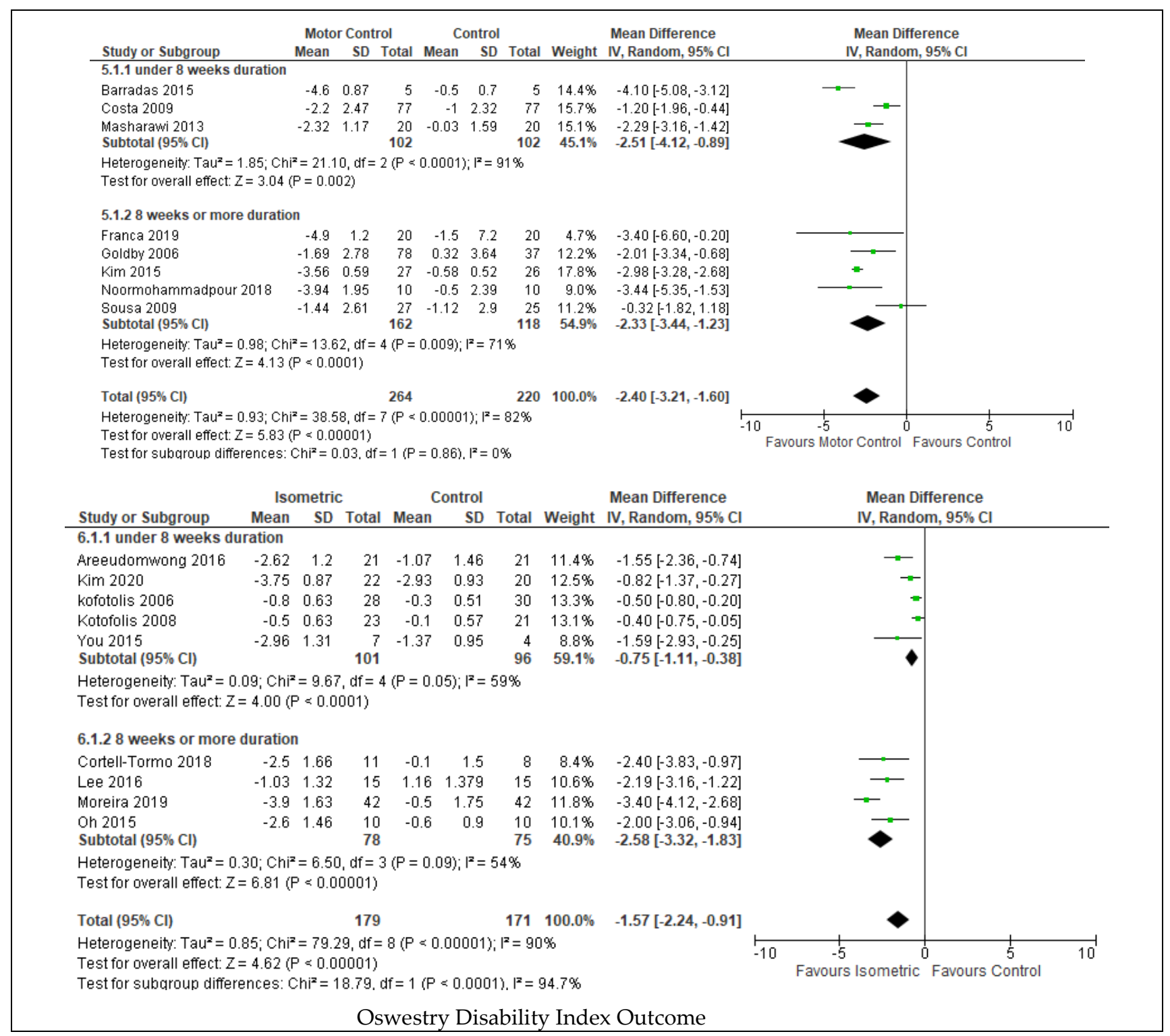




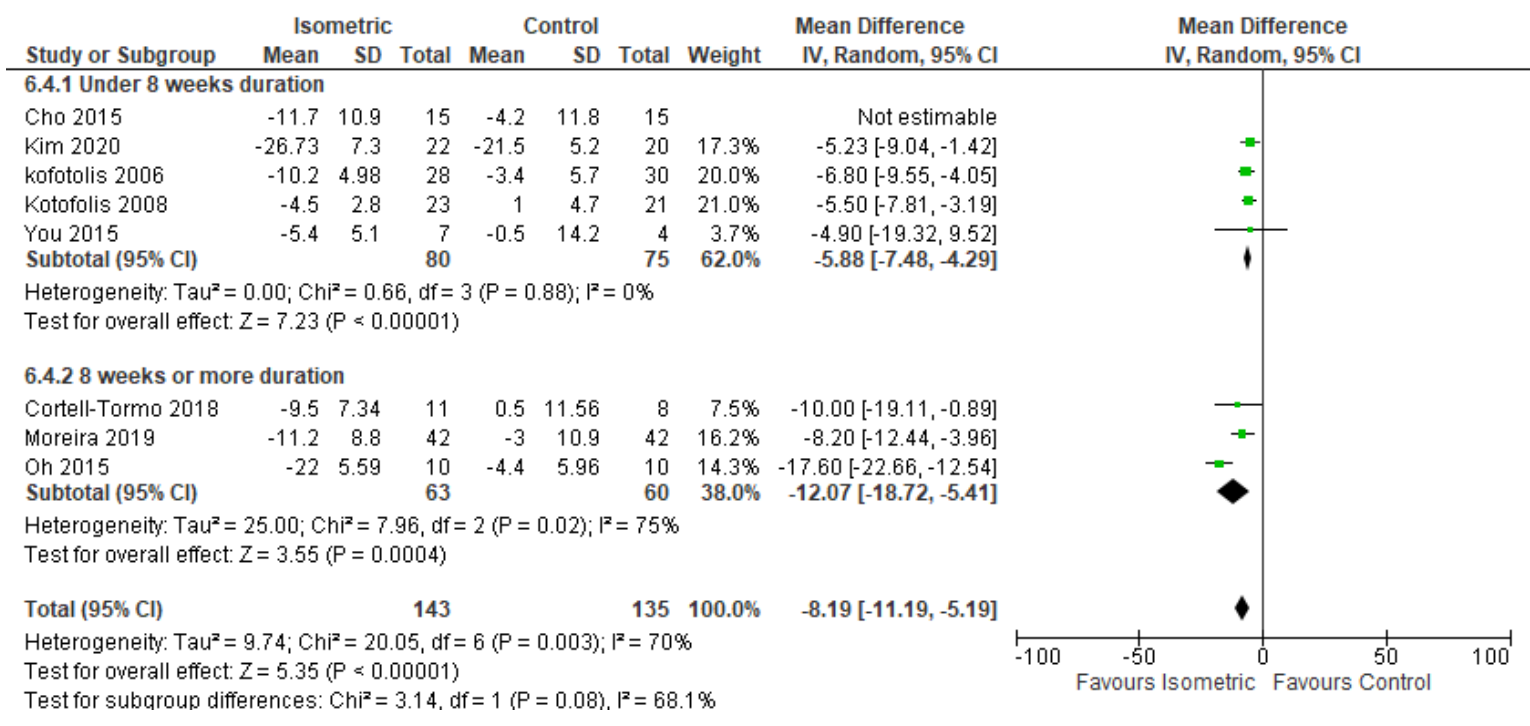

Appendix C.3. Subgroup Analysis of Isometric and Motor Control Interventions Based on Mean Age

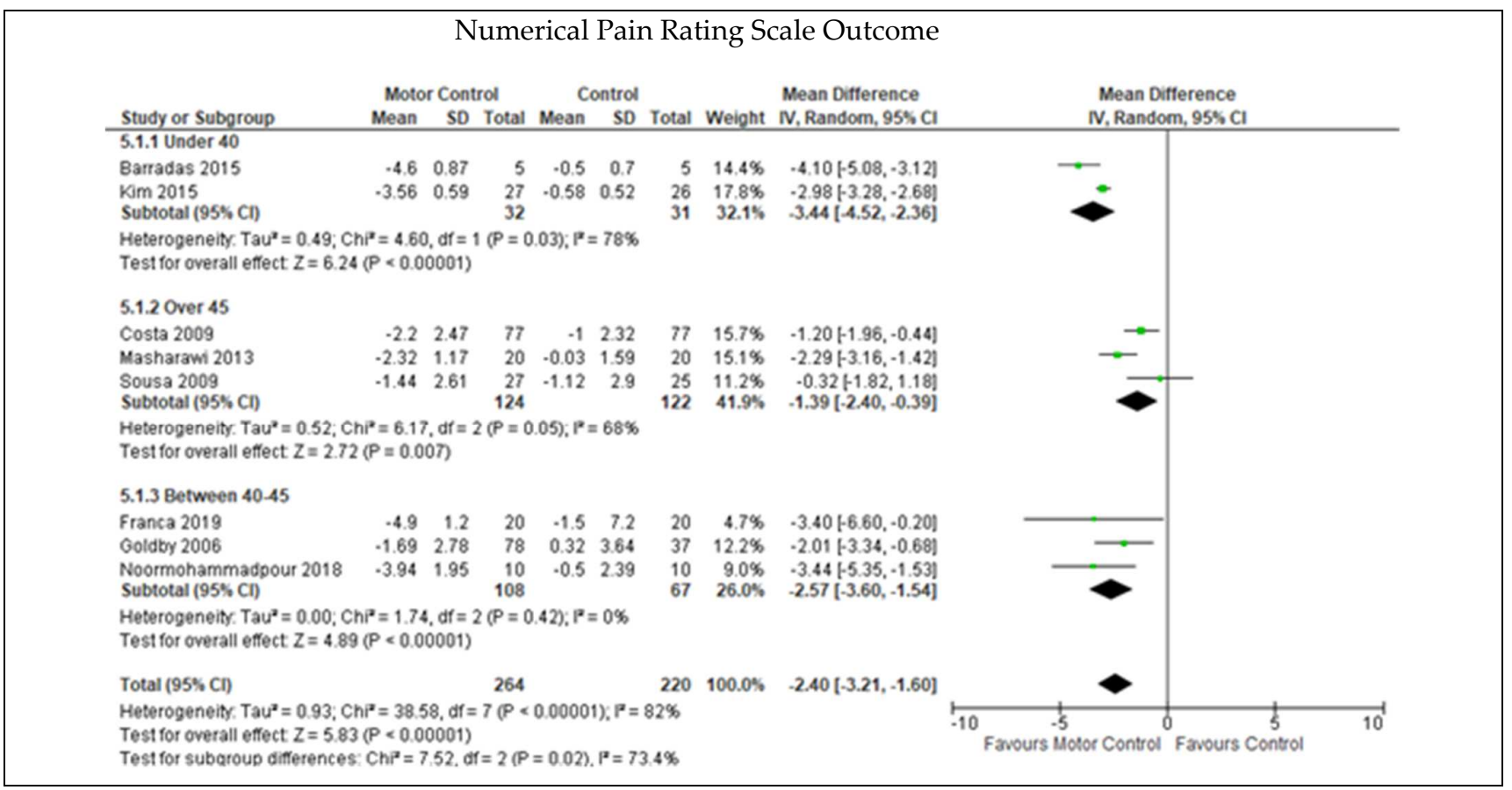




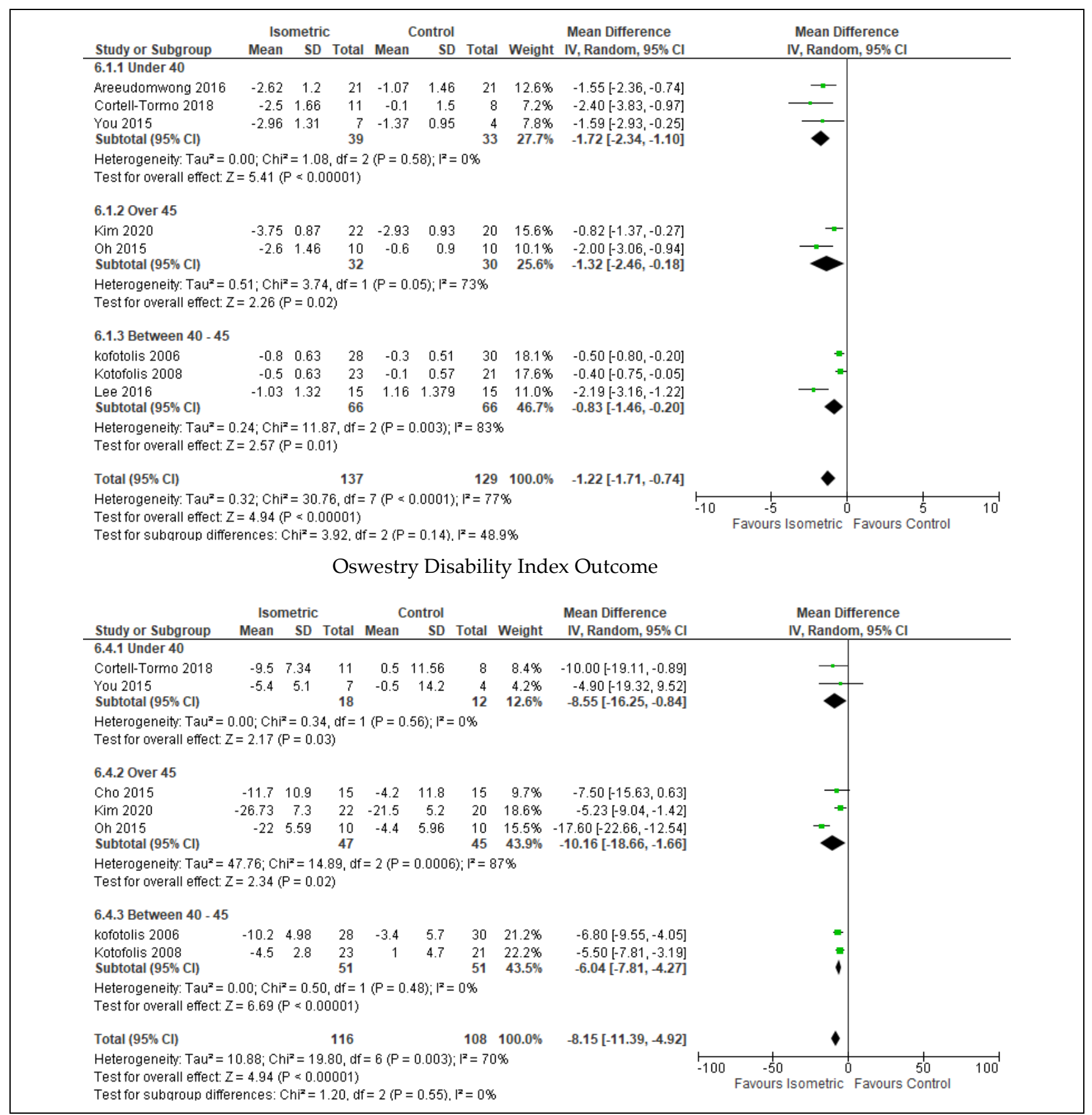

\section{References}

1. Deyo, R.A.; Rainville, J.; Kent, D.L. What can the history and physical examination tell us about low back pain? JAMA 1992, 268, 760-765. [CrossRef] [PubMed]

2. $\quad$ Freburger, J.K.; Holmes, G.M.; Agans, R.P.; Jackman, A.M.; Darter, J.D.; Wallace, A.S.; Castel, L.D.; Kalsbeek, W.D.; Carey, T.S. The rising prevalence of chronic low back pain. Arch. Intern. Med. 2009, 169, 251-258. [CrossRef] [PubMed]

3. Airaksinen, O.; Brox, J.I.; Cedraschi, C.; Hildebrandt, J.; Klaber-Moffett, J.; Kovacs, F.; Mannion, A.F.; Reis, S.H.M.U.E.L.; Staal, J.B.; Ursin, H.; et al. Chapter 4 European guidelines for the management of chronic nonspecific low back pain. Eur. Spine J. 2006, 15, s192-s300. [CrossRef]

4. Deyo, R.A.; Mirza, S.K.; Martin, B.I. Back pain prevalence and visit rates: Estimates from U.S. national surveys, 2002. Spine 2006, 31, 2724-2727. [CrossRef] [PubMed]

5. Wong, W.S.; Fielding, R. Prevalence and Characteristics of Chronic Pain in the General Population of Hong Kong. J. Pain 2011, 12, 236-245. [CrossRef] [PubMed] 
6. Cholewicki, J.; Juluru, K.; McGill, S.M. Intra-abdominal pressure mechanism for stabilizing the lumbar spine. J. Biomech. 1999, 32, 13-17. [CrossRef]

7. Gunning, J.; Callaghan, J.; McGill, S. The role of prior loading history and spinal posture on the compressive tolerance and type of failure in the spine using a porcine trauma model. Clin. Biomech. 2001, 16, 471-480. [CrossRef]

8. Veres, S.P.; Robertson, P.A.; Broom, N.D. The morphology of acute disc herniation: A clinically relevant model defining the role of flexion. Spine 2009, 34, 2288-2296. [CrossRef]

9. Yingling, V.R.; McGill, S.M. Anterior shear of spinal motion segments: Kinematics, kinetics, and resultant injuries observed in a porcine model. Spine 1999, 24, 1882. [CrossRef]

10. Gore, M.; Sadosky, A.; Stacey, B.R.; Tai, K.S.; Leslie, D. The burden of chronic low back pain: Clinical comorbidities, treatment patterns, and health care costs in usual care settings. Spine 2012, 37, E668-E677. [CrossRef]

11. McGill, S.M.; Childs, A.; Liebenson, C. Endurance times for low back stabilization exercises: Clinical targets for testing and training from a normal database. Arch. Phys. Med. Rehabil. 1999, 80, 941-944. [CrossRef]

12. O'Sullivan, P.B.; Twomey, L.T.; Allison, G.T. Evaluation of specific stabilizing exercise in the treatment of chronic low back pain with radiologic diagnosis of spondylolysis of spondylolisthesis. Spine 1997, 22, 2959-2967. [CrossRef]

13. Zatsiorsky, V.M. Science and Practice of Strength Training, 2nd ed.; Human Kinetics: Champaign, IL, USA, 2006.

14. Axler, C.T.; McGill, S.M. Low back loads over a variety of abdominal exercises: Searching for the safest abdominal challenge. Med. Sci. Sports Exerc. 1997, 29, 804-811. [CrossRef] [PubMed]

15. Cohen, I.; Rainville, J. Aggressive Exercise as Treatment for Chronic Low Back Pain. Sports Med. 2002, 32, 75-82. [CrossRef] [PubMed]

16. Moseley, L. Combined physiotherapy and education is efficacious for chronic low back pain. Aust. J. Physiother. 2002, 48, 297-302. [CrossRef]

17. Maeo, S.; Takahashi, T.; Takai, Y.; Kanehisa, H. Trunk muscle activities during abdominal bracing: Comparison among muscles and exercises. J. Sports Sci. Med. 2013, 12, 467-474. [PubMed]

18. Tampier, C.; Drake, J.D.; Callaghan, J.P.; McGill, S.M. Progressive disc herniation: An investigation of the mechanism using radiologic, histochemical, and microscopic dissection techniques on a porcine model. Spine 2007, 32, 2869-2874. [CrossRef]

19. McGill, S. Low Back Disorders: Evidence-Based Prevention and Rehabilitation, 3rd ed.; Human Kinetics: Champaign, IL, USA, 2016.

20. Rathod, S.; Shah, N. The Effect of Training Core Stabilizers in Clerks with Low Back Pain. Indian J. Physiother. Occup. Ther. 2015, 9 , 141-146. [CrossRef]

21. Calatayud, J.; Guzman-Gonzalez, B.; Andersen, L.L.; Cruz-Montecinos, C.; Morell, M.T.; Roldán, R.; Ezzatvar, Y.; Casaña, J. Effectiveness of a group-based progressive strength training in primary care to improve the recurrence of low back pain exacerbations and function: A randomised trial. Int. J. Environ. Res. Public Health 2020, 17, 8326. [CrossRef]

22. Akhtar, M.W.; Karimi, H.; Gilani, S.A. Effectiveness of core stabilization exercises and routine exercise therapy in management of pain in chronic nonspecific low back pain: A randomized controlled clinical trial. Pak. J. Med. Sci. 2017, 33, 1002-1006. [CrossRef]

23. Bae, C.R.; Jin, Y.; Yoon, B.C.; Kim, N.H.; Park, K.W.; Lee, S.H. Effects of assisted sit-up exercise compared to core stabilization exercise on patients with non-specific low back pain: A randomized controlled trial. J. Back Musculoskelet. Rehabil. 2018, 31, 871-880. [CrossRef] [PubMed]

24. Santaella da Fonseca Lopes de Sousa, K.; Orfale, A.G.; Meireles, S.M.; Roberto Leite, J.; Natour, J. Assessment of a biofeedback program to treat chronic low back pain. J. Musculoskelet. Pain 2009, 17, 369-377. [CrossRef]

25. Masharawi, Y.; Nadaf, N. The effect of non-weight bearing group-exercising on females with non-specific chronic low back pain: A randomized single blind controlled pilot study. J. Back Musculoskelet. Rehabil. 2013, 26, 353-359. [CrossRef] [PubMed]

26. Hayden, J.; Van Tulder, M.W.; Malmivaara, A.; Koes, B.W. Exercise therapy for treatment of non-specific low back pain. Cochrane Database Syst. Rev. 2005. [CrossRef] [PubMed]

27. Smith, B.E.; Littlewood, C.; May, S. An update of stabilisation exercises for low back pain: A systematic review with meta-analysis. BMC Musculoskelet. Disord. 2014, 15, 1-21. [CrossRef] [PubMed]

28. Owen, P.J.; Miller, C.T.; Mundell, N.L.; Verswijveren, S.J.; Tagliaferri, S.D.; Brisby, H.; Bowe, S.J.; Belavy, D.L. Which specific modes of exercise training are most effective for treating low back pain? Network meta-analysis. Br. J. Sports Med. 2020, 54, 1279-1287. [CrossRef]

29. Davies, C.C.; Nitz, A.J. Psychometric properties of the Roland-Morris Disability Questionnaire compared to the Oswestry Disability Index: A systematic review. Phys. Ther. Rev. 2009, 14, 399-408. [CrossRef]

30. Rethlefsen, M.L.; Kirtley, S.; Waffenschmidt, S.; Lakner, Z.; Popp, J. PRISMA-S: An extension to the PRISMA Statement for Reporting Literature Searches in Systematic Reviews. Syst. Rev. 2021, 10, 39. [CrossRef] [PubMed]

31. Parreira, P.; Heymans, M.W.; van Tulder, M.W.; Esmail, R.; Koes, B.W.; Poquet, N.; Lin, C.W.C.; Maher, C.G. Back Schools for chronic non-specific low back pain. Cochrane Database Syst. Rev. 2017. [CrossRef]

32. Hildebrandt, V.H. Back pain in the working population: Prevalence rates in Dutch trades and professions. Ergonomics 1995, 38, 1283-1298. [CrossRef] [PubMed]

33. Kofotolis, N.; Kellis, E. Effects of two 4-week proprioceptive neuromuscular facilitation programs on muscle endurance, flexibility, and functional performance in women with chronic low back pain. Phys. Ther. 2006, 86, 1001-1012. [CrossRef] [PubMed]

34. Aasa, B.; Berglund, B.; Aasa, U. Potential predictors of activity and pain intensity twelve months after spinal motor control training in patients with low back pain. Physiotherapy 2015, 1, eS26-eS27. [CrossRef] 
35. Qaseem, A.; Wilt, T.J.; McLean, R.M.; Forciea, M.A. Clinical Guidelines Committee of the American College of Physicians Noninvasive Treatments for Acute, Subacute, and Chronic Low Back Pain: A Clinical Practice Guideline From the American College of Physicians. Ann. Intern. Med. 2017, 166, 514. [CrossRef]

36. Nadeau, M.; Rosas-Arellano, M.P.; Gurr, K.R.; Bailey, S.I.; Taylor, D.C.; Grewal, R.; Lawlor, D.K.; Bailey, C.S. The reliability of differentiating neurogenic claudication from vascular claudication based on symptomatic presentation. Can. J. Surg. 2013, 56, 372. [CrossRef]

37. Ross, M.D.; Bayer, E. Cancer as a cause of low back pain in a patient seen in a direct access physical therapy setting. J. Orthop. Sports Phys. Ther. 2005, 35, 651-658. [CrossRef] [PubMed]

38. Staud, R. Evidence for shared pain mechanisms in osteoarthritis, low back pain, and fibromyalgia. Curr. Rheumatol. Rep. 2011, 13, 513. [CrossRef]

39. Hussain, N.S.; Perez-Cruet, M.J. Complication management with minimally invasive spine procedures. Neurosurg. Focus 2011, 31, E2. [CrossRef]

40. Menon, S.K.; Onyia, C.U. A short review on a complication of lumbar spine surgery: CSF leak. Clin. Neurol. Neurosurg. 2015, 139, 248-251. [CrossRef] [PubMed]

41. Gladwell, V.; Head, S.; Haggar, M.; Beneke, R. Does a program of Pilates improve chronic non-specific low back pain? 449. J. Sport Rehabil. 2006, 15, 338-350. [CrossRef]

42. Abass, A.O.; Alli, A.R.; Olagbegi, O.M.; Christie, C.J.; Bolarinde, S.O. Effects of an eight-week lumbar stabilization exercise programme on selected variables of patients with chronic low back pain. Bangladesh J. Med. Sci. 2020, 19, 467-474. [CrossRef]

43. Wewege, M.A.; Booth, J.; Parmenter, B.J. Aerobic vs. resistance exercise for chronic non-specific low back pain: A systematic review and meta-analysis. J. Back Musculoskelet. Rehabil. 2018, 31, 889-899. [CrossRef] [PubMed]

44. Khadilkar, A.; Odebiyi, D.O.; Brosseau; Wells, G.A. Transcutaneous electrical nerve stimulation (TENS) versus placebo for chronic low-back pain. Cochrane Database Syst. Rev. 2008. [CrossRef] [PubMed]

45. Ebadi, S.; Henschke, N.; Forogh, B.; Ansari, N.N.; van Tulder, M.W.; Babaei-Ghazani, A.; Fallah, E. Therapeutic ultrasound for chronic low back pain. Cochrane Database Syst. Rev. 2020. [CrossRef]

46. Steffens, D.; Maher, C.G.; Pereira, L.S.; Stevens, M.L.; Oliveira, V.C.; Chapple, M.; Teixeira-Salmela, L.F.; Hancock, M.J. Prevention of low back pain: A systematic review and meta-analysis. JAMA Intern. Med. 2016, 176, 199-208. [CrossRef] [PubMed]

47. Biering-Sørensen, F. Physical measurements as risk indicators for low-back trouble over a one-year period. Spine 1984, 9, 106-119. [CrossRef] [PubMed]

48. Denteneer, L.; Van Daele, U.; Truijen, S.; De Hertogh, W.; Meirte, J.; Stassijns, G. Reliability of physical functioning tests in patients with low back pain: A systematic review. Spine J. 2018, 18, 190-207. [CrossRef] [PubMed]

49. Del Pozo-Cruz, B.; Mocholi, M.H.; del Pozo-Cruz, J.; Parraca, J.A.; Adsuar, J.C.; Gusi, N. Reliability and validity of lumbar and abdominal trunk muscle endurance tests in office workers with nonspecific subacute low back pain. J. Back Musculoskelet. Rehabil. 2014, 27, 399-408. [CrossRef] [PubMed]

50. Pilz, B.; Vasconcelos, R.A.; Teixeira, P.P.; Mello, W.; Oliveira, I.O.; Ananias, J.; Timko, M.; Grossi, D.B. Comparison of Hip and Lumbopelvic Performance Between Chronic Low Back Pain Patients Suited for the Functional Optimization Approach and Healthy Controls. Spine 2020, 45, E37-E44. [CrossRef] [PubMed]

51. Chiarotto, A.; Boers, M.; Deyo, R.A.; Buchbinder, R.; Corbin, T.P.; Costa, L.O.; Foster, N.E.; Grotle, M.; Koes, B.W.; Kovacs, F.M.; et al. Core outcome measurement instruments for clinical trials in nonspecific low back pain. Pain 2018, 159, 481. [CrossRef] [PubMed]

52. Smeets, R.; Köke, A.; Lin, C.W.; Ferreira, M.; Demoulin, C. Measures of function in low back pain/disorders: Low back pain rating scale (LBPRS), oswestry disability index (ODI), progressive isoinertial lifting evaluation (PILE), quebec back pain disability scale (QBPDS), and roland-morris disability questionnaire (RDQ). Arthritis Care Res. 2011, 63, S158-S173.

53. Savigny, P.; Watson, P.; Underwood, M. Early management of persistent non-specific low back pain: Summary of NICE guidance. BMJ 2009, 338. [CrossRef]

54. Liguori, G.; American College of Sports Medicine. ACSM's guidelines for exercise testing and prescription. In American College of Sports Medicine's Guidelines for Exercise Testing and Prescription, 11th ed.; Wolters Kluwer: Philadelphia, PA, USA, 2021.

55. Higgins, J.P.; Thompson, S.G.; Deeks, J.J.; Altman, D.G. Measuring inconsistency in meta-analyses. BMJ 2003, 327, 557-560. [CrossRef] [PubMed]

56. Sterne, J.A.; Savović, J.; Page, M.J.; Page, M.J.; Hróbjartsson, A.; Boutron, I.; Reeves, B.; Eldridge, S. RoB 2: A revised tool for assessing risk of bias in randomised trials. BMJ 2019, 366. [CrossRef] [PubMed]

57. Suni, J.; Rinne, M.; Natri, A.; Statistisian, M.P.; Parkkari, J.; Alaranta, H. Control of the lumbar neutral zone decreases low back pain and improves self-evaluated work ability: A 12-month randomized controlled study. Spine 2006, 31, E611-E620. [CrossRef] [PubMed]

58. Hicks, G.E.; Fritz, J.M.; Delitto, A.; McGill, S.M. Preliminary Development of a Clinical Prediction Rule for Determining Which Patients With Low Back Pain Will Respond to a Stabilization Exercise Program. Arch. Phys. Med. Rehabil. 2005, 86, 1753-1762. [CrossRef] [PubMed]

59. Fors, M.; Enthoven, P.; Abbott, A.; Öberg, B. Effects of pre-surgery physiotherapy on walking ability and lower extremity strength in patients with degenerative lumbar spine disorder: Secondary outcomes of the PREPARE randomised controlled trial. BMC Musculoskelet. Disord. 2019, 20, 468. [CrossRef] 
60. Puntumetakul, R.; Chalermsan, R.; Hlaing, S.S.; Tapanya, W.; Saiklang, P.; Boucaut, R. The effect of core stabilization exercise on lumbar joint position sense in patients with subacute non-specific low back pain: A randomized controlled trial. J. Phys. Ther. Sci. 2018, 30, 1390-1395. [CrossRef] [PubMed]

61. Storheim, K.; Brox, J.I.; Holm, I.; Koller, A.K.; Bo, K. Intensive group training versus cognitive intervention in sub-acute low back pain: Short-term results of a single-blind randomized controlled trial. J. Rehabil. Med. 2003, 35, 132-140. [CrossRef] [PubMed]

62. Wong, E.Y.; Deyo, R.A. Acute low back pain. Prim. Care Update OB/GYNS 2001, 8, 171-174. [CrossRef]

63. Günay, S.; Yildirim, Y.; Karadibak, D. The effect of the muscle endurance training on the chronic low back pain. Türk Fizyoterapi ve Rehabilitasyon Dergisi/Turkish. J. Physiother. Rehabil. 2014, 25, 4. [CrossRef]

64. Gonzalez-Medina, G.; Perez-Cabezas, V.; Ruiz-Molinero, C.; Chamorro-Moriana, G.; Jimenez-Rejano, J.J.; Galán-Mercant, A. Effectiveness of Global Postural Re-Education in Chronic Non-Specific Low Back Pain: Systematic Review and Meta-Analysis. J. Clin. Med. 2021, 10, 5327. [CrossRef] [PubMed]

65. Ikeda, D.M.; McGill, S.M. Can altering motions, postures, and loads provide immediate low back pain relief: A study of 4 cases investigating spine load, posture, and stability. Spine 2012, 37, E1469-E1475. [CrossRef] [PubMed]

66. Merinero, D.; Rodríguez-Aragón, M.; Álvarez-González, J.; López-Samanes, Á.; López-Pascual, J. Acute Effects of Global Postural Re-Education on Non-Specific Low Back Pain. Does Time-of-Day Play a Role? Int. J. Environ. Res. Public Health 2021, 18, 713. [CrossRef] [PubMed]

67. Shamsi, M.; Mirzaei, M.; HamediRad, M. Comparison of muscle activation imbalance following core stability or general exercises in nonspecific low back pain: A quasi-randomized controlled trial. BMC Sports Sci. Med. Rehabil. 2020, 12, 1-9. [CrossRef] [PubMed]

68. Shamsi, M.B.; Rezaei, M.; Zamanlou, M.; Sadeghi, M.; Pourahmadi, M.R. Does core stability exercise improve lumbopelvic stability (through endurance tests) more than general exercise in chronic low back pain? A quasi-randomized controlled trial. Physiother. Theory Pract. 2016, 32, 171-178. [CrossRef] [PubMed]

69. Shamsi, M.B.; Sarrafzadeh, J.; Jamshidi, A. Comparing core stability and traditional trunk exercise on chronic low back pain patients using three functional lumbopelvic stability tests. Physiother. Theory Pract. 2015, 31, 89-98. [CrossRef]

70. Koumantakis, G.A.; Watson, P.J.; Oldham, J.A. Trunk muscle stabilization training plus general exercise versus general exercise only: Randomized controlled trial of patients with recurrent low back pain. Phys. Ther. 2005, 85, 209-225. [CrossRef]

71. Majiwala, B.A.; Warude, T.A.; Pawar, A. Effect of isometric and isotonic exercise training on core muscle in patients with non-specific low back pain. Asian J. Pharm. Clin. Res. 2017, 10, 308-311. [CrossRef]

72. Bickel, C.S.; Cross, J.M.; Bamman, M.M. Exercise dosing to retain resistance training adaptations in young and older adults. Med. Sci. Sports Exerc. 2011, 43, 1177-1187. [CrossRef] [PubMed]

73. Moher, D.; Hopewell, S.; Schulz, K.F.; Montori, V.; Gøtzsche, P.C.; Devereaux, P.J.; Elbourne, D.; Egger, M.; Altman, D.G. CONSORT 2010 Explanation and Elaboration: Updated guidelines for reporting parallel group randomised trials. J. Clin. Epidemiol. 2010, 63, e1-e37. [CrossRef] [PubMed] 